\title{
Growth of Metal Clusters at Surfaces
}

\author{
Harald Brune
}

\section{Introduction}

In the present chapter we will discuss the creation of surface supported metal clusters through nucleation and growth in Molecular Beam Epitaxy (MBE). MBE is commonly used to grow thin epitaxial films from the vapor phase onto single crystal substrates under ultra-high vacuum (UHV) conditions [1]. In thin film growth the nucleation of stable clusters is the preliminary step to the immobilization and further condensation of film atoms or molecules. If one is close to thermodynamic equilibrium it is irrelevant how and where this nucleation takes place and the film topography is given by the balance of the free energies of film surface, substrate surface, and the interface between the two [2]. However, the prerequisite to the growth is to be away from thermodynamic equilibrium, at least to some extent, since detailed balance arguments require that in equilibrium all processes appear with equal rates, including condensation and desorption. Therefore, the entire growth system hardly adopts equilibrium and is thus in a state always influenced to a certain extent by kinetics. The history of when and where the film atoms were added begins to matter and the film morphology is determined by the microscopic pathway taken by the system. This pathway comprises the interplay of only a few elementary processes, such as terrace diffusion of single adatoms, cluster formation and diffusion, as well as aggregation and interlayer diffusion. All diffusion events take place on a time scale set by the coverage divided by the deposition flux. The importance of kinetics in thin film growth was realized early on, leading to the development of mean-field nucleation theory which relates the cluster density to monomer and cluster diffusion rates and to cluster dissociation rates [3-5]. Continued interest in the elementary processes of epitaxial growth has led to the quantification of energy barriers for the most important of these processes. On the experimental side techniques such as Field Ion Microscopy (FIM) [6-9] and by Variable Temperature Scanning Tunneling Microscopy (VT-STM) [10] have been used. On the theoretical side the barriers for these processes were evaluated using approximative methods such as the Embedded Atom Method (EAM) or Effective Medium Theory (EMT) and ab initio theoretical concepts based on Density Functional Theory (DFT). The study of the elementary processes involved in 
epitaxial growth and their relation to cluster and film morphology are topics of ongoing interest.

The knowledge gained from such surface science studies can be employed to grow clusters at surfaces with well defined size, shape and even regular spacing. The idea of assembling supported clusters from adatoms might appear unusual to readers belonging to the cluster community, as typically in cluster physics, clusters are condensed, cooled and mass selected in the gas phase before they eventually become (soft-) landed onto the support. The aim of the present chapter is to convince the reader of the high degree of control on cluster size, shape and spacing that can be achieved in the MBE growth of clusters at single crystal surfaces. Of course, the attempt to grow well defined structures at specific sites is a struggle against the statistics inherent in deposition and in the Brownian character of thermally activated adatom diffusion. The statistics of both processes expresses itself in the clusters' spatial and size distributions. These distributions are coupled to each other and their width and shape are given by well known scaling laws of nucleation [11-14]. However, we will show below that there are means to overcome statistical limitations and, to some extent, to create order out of randomness.

The chapter is organized as follows. We will start with an introduction to the elementary processes of epitaxial growth. In the following Sects. 3-5 we discuss cluster growth on isotropic metal surfaces. First we give a brief outline of the basic results of nucleation theory in its simplest form of stable and immobile dimers. We show experiments that confirm the theory for that case and permit a direct link between nucleation densities and terrace diffusion parameters. In Sect. 4 we discuss the transitions from fractal to various compact clusters tracing back the cluster shape to the atomic processes of aggregation. The last section on isotropic substrates is devoted to Ostwald ripening as a means of preparing compact two-dimensional (2D) clusters with a narrow size distribution centered at almost any desired value. Section 6 discusses cluster growth on anisotropic metal substrates where diffusion and/or sticking anisotropy give rise to the formation of elongated clusters, and in the extreme case to 1D atomic chains. In the following section we turn to surfaces with dislocation networks or Moiré patterns. Such substrates provide inhomogeneous potential energy surfaces that may guide diffusing monomers to singular sites where they nucleate clusters. The result is a periodic cluster array, the regularity in spacing being accompanied by narrow cluster size distributions. The nucleation of metal clusters on single crystal oxides and sulfides is discussed in Sect. 8. We will end this chapter with a brief conclusion and outlook.

\section{The Elementary Processes of MBE Growth}

In molecular beam epitaxy, film atoms are deposited onto the substrate with thermal energy $(\sim 0.1 \mathrm{eV})$ and flux $F$ (expressed in atoms per lattice site, 
equivalent to monolayers (ML), per second). Typically, the energy gained in the adsorbate-substrate bond formation is effectively dissipated into the lattice, so that adatoms are brought into thermal equilibrium with the substrate already at their site of impact [15]. This can be inferred for instance from experiments at low temperatures revealing cluster sizes in agreement with the predictions of statistical growth, allowing no diffusion at all [10,16-18]. Therefore transient jumps are the exception, if they exist at all for metal on metal adsorption. (In contrast, there is evidence for transient motion for the dissociative chemisorption of molecules on metals [19,20].) Transient nonthermal motion has to be distinguished from thermal motion with small energy barriers towards next nearest neighbors [21] and clusters [22,23]. For certain combinations of elements the adsorption energy might be used to trigger transient exchange processes, even at low $T$ [24]. However, in the absence of exchange and sufficiently far away from clusters or adatoms, the film atoms generally adsorb where they land from the vapor phase. From there on, adatom diffusion sets in.

The diffusion processes participating in epitaxial growth are thermally activated jumps mostly in the form of straight adatom movements between adjacent lattice sites. However, concerted motion of several atoms may also be involved. Transition State Theory (TST) [25] assumes that the atoms stay between two subsequent jumps long enough in their adsorption wells to thermally equilibrate, in addition, recrossing of the barrier is assumed to be negligible. These assumptions are justified if the energy barrier separating the binding from the transition site satisfies $E \ll k_{\mathrm{B}} T$. The jump rate of a diffusion process of type $n$ is then given by Boltzmann statistics as $\nu_{n}=$ $\nu_{0, n} \exp \left(-E_{n} / k_{\mathrm{B}} T\right)$, with the attempt frequency $\nu_{0, n}$ typically being in the range $10^{12}-10^{13} \mathrm{~Hz}$ of Debye frequencies.

Terrace migration of single adatoms $\left(E_{\mathrm{m}}\right.$ in Fig. 1$)$ is the most fundamental of these diffusion processes. It gives rise to nucleation of islands on substrate terraces or to step flow growth at elevated temperatures. Depending on the density of simultaneously diffusing particles, one distinguishes the collective diffusion coefficient (also known as chemical or Fickian diffusion coefficient) of an ensemble of mutually interacting particles from the tracer (or intrinsic) diffusion coefficient describing the mean square displacement of one isolated random walker per unit time [26]. For typical growth rates the density of diffusing particles is rather small (the monomer density $n_{1}<10^{-3}$ adatoms per adsorption site). The mean inter particle distance is therefore large compared to typical interaction ranges for metal adatoms on metal substrates $[27,28]$ and cluster densities are determined by the tracer diffusion coefficient $D$ defined as

$$
D=D_{0} \exp \left(-E_{\mathrm{m}} / k_{\mathrm{B}} T\right), \text { with } D_{0}=\frac{1}{4} \nu_{0},
$$

where $D$ is expressed in substrate unit cells per second. The factor of $1 / 4$ in $D_{0}$ is valid for $2 \mathrm{D}$ diffusion, whilst in one dimension this factor is $1 / 2$. 


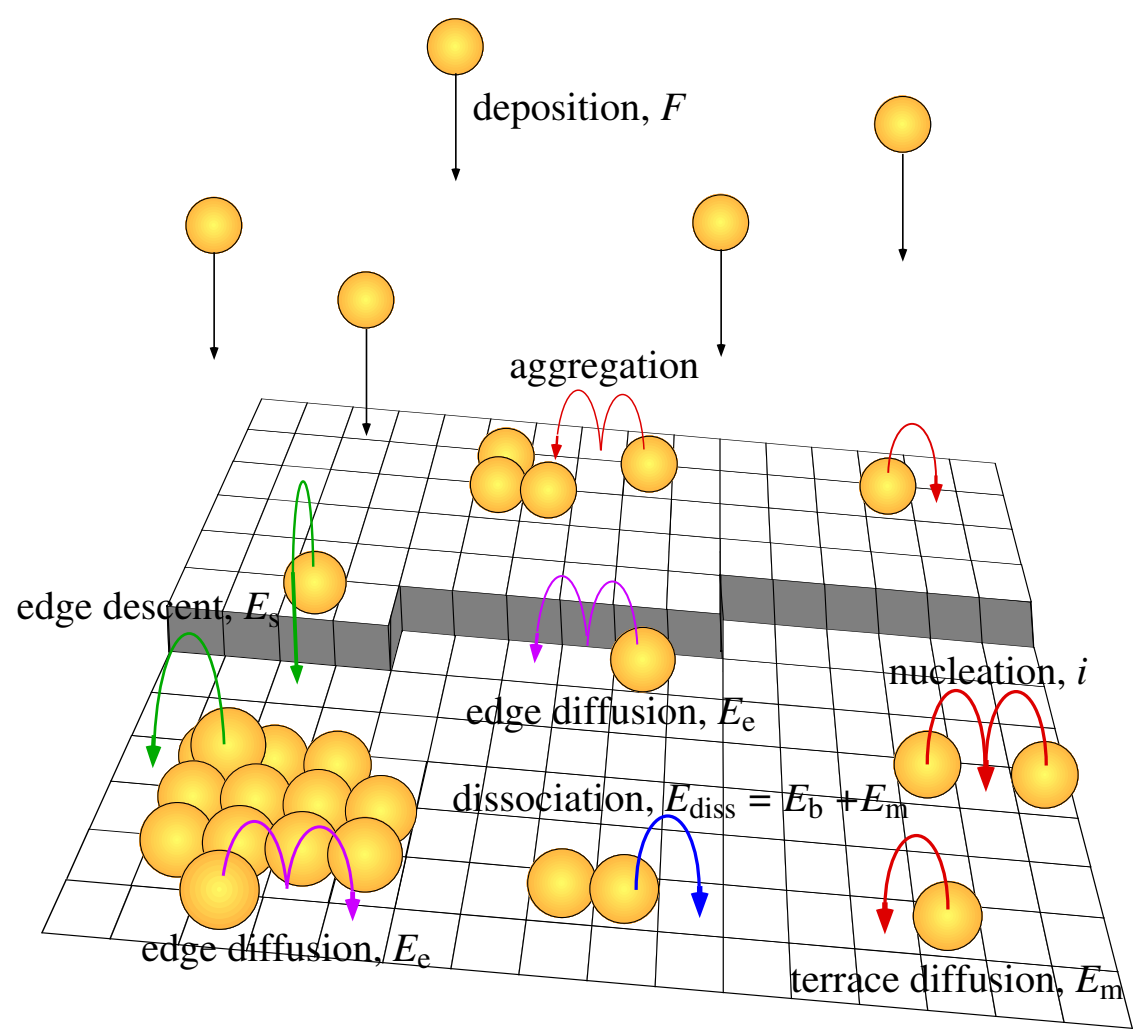

Fig. 1. The elementary diffusion processes of MBE growth

Diffusion across the terrace ends when the adatoms collide with one or more of their own (for sake of simplicity structural and chemical defects are not considered). Depending on the lateral bond energy ( $E_{\mathrm{b}}$ in Fig. 1) and the number of neighbors, the formed cluster remains stable or decays again. A stable nucleus is a cluster that is large enough to grow more rapidly than it decays on the time-scale of deposition. One defines the critical cluster size $i$ by the number of atoms in the smallest stable nucleus minus one, i.e., attachment of one atom turns a critical cluster into a stable one.

The two-dimensional cluster shape is determined by the mobility of aggregating adatoms along the cluster edge (barrier $E_{\mathrm{e}}$ in Fig. 1), more specifically along straight steps and around kinks and corners, in the case of trigonal substrates also from 1-fold coordinated corner to 2 -fold coordinated step sites. Low mobility leads to ramified clusters with fractal dimension, similar to Diffusion Limited Aggregation (DLA) scenarios [29-31]. Anisotropic terrace diffusion imposed by the substrate symmetry, in conjunction with anisotropic sticking to the edges, can lead to the formation of 1D monoatomic wires. Com- 
pact 2D clusters are created if edge diffusion is fast compared to the cluster growth rate. The thermodynamic $2 \mathrm{D}$ equilibrium shape forms when corner and kink crossing become activated.

The rate of adatom descent at cluster steps determines whether or not adatoms deposited onto the cluster top are able to descend to the substrate level before other adatoms become deposited onto the cluster top. In the latter case a stable nucleus forms on top of the cluster giving rise to the kinetic growth of 3D clusters, whereas in the first case clusters remain 2D until they coalesce. An adatom approaching a descending step encounters a barrier $E_{\mathrm{s}}$ for descent which is typically larger than $E_{\mathrm{m}}$. The extra diffusion barrier to overcome atomic steps was experimentally discovered [32] and theoretically conjectured [33] long ago. It is caused by the strongly reduced coordination of the adatom to the substrate in the transition state, or in the case of exchange interlayer diffusion, by the reduced coordination of the complex transition state configuration. Ab initio calculations give insight into why exchange diffusion is preferred for specific step orientations and combinations of metallic elements whereas for other step orientations and systems interlayer diffusion is a simple roll down process. The values for $E_{\mathrm{s}}$ derived from such calculations [34-36] can be compared to results from FIM experiments $[15,37]$. Experiment and theory agree that the mechanism of interlayer diffusion is strongly system specific. Complementary to direct FIM inspection there are also various indirect ways to infer experimental estimates on $E_{\mathrm{s}}$ from layer occupation numbers $[38,39]$, from the nucleation probability on cluster tops [40], from island decay in suitable geometries [41], or from step densities $[42,43]$ and slopes of mounds evolving through kinetic roughening [44,45]. The values of $E_{\mathrm{s}}$ derived from such observations of the film morphology are all effective barriers for interlayer diffusion. Despite their relevance for predicting the epitaxial growth morphology and cluster dimension, association to a particular microscopic interlayer diffusion process is often not unambiguously possible. For sake of simplicity the following discussion of metal on metal systems will be restricted to 2D islands only, i.e., the STM images below show monolayer high islands. For kinetically caused 3D island growth the reader is referred to the references given in this paragraph.

\section{Nucleation}

We will now address the relationship between cluster density and terrace diffusion coefficient, cluster binding energy and deposition flux. For simplicity we discuss the case of $2 \mathrm{D}$ islands in the irreversible growth regime where the critical cluster is a monomer, i.e., $i=1$, and a dimer is stable and immobile. For an extension of this discussion to more complicated cases of larger critical cluster sizes, 3D clusters, incomplete condensation, or cluster diffusion, we refer to $[4,46-48]$. 
The simplest MBE growth scenario has monomers as the only mobile species and dimers represent stable nuclei. In the initial phase of deposition the adatoms arriving from the gas phase with rate $F$ diffuse with rate $D$ on the substrate terraces until they meet a second diffusing adatom and create a dimer. As deposition proceeds, the number of dimers will increase linearly until their density $n_{2}$ becomes comparable to the monomer density $n_{1}$. From there on, the probabilities that a diffusing monomer encounters one of its own or a dimer become comparable and the growth of stable clusters starts to compete with the nucleation of new ones. As a consequence, the increase in density of stable nuclei $n_{x}$ ( $x$ standing for any size that is stable, $x \geq 2$ ) levels off until $n_{x}$ saturates at a coverage of typically $\theta_{\text {sat }} \sim 0.15 \mathrm{ML}$. When the saturation island density is reached, the mean free path of diffusing adatoms is equal to the mean island separation and any further deposition will exclusively lead to island growth since all adatoms reach and attach themselves to existing islands. At coverages beyond $0.2 \mathrm{ML}$ the $2 \mathrm{D}$ clusters start to coalesce until the monolayer film percolates at typically $\theta=0.5 \mathrm{ML}$. For metal on metal growth this scenario was experimentally verified for $\mathrm{Ag} / \mathrm{Pt}(111)$ by means of VT-STM [49]. The STM observations ranged from the pure nucleation phase with its linear increase of $n_{x}$ and an average cluster size of 2-3 atoms up to saturation and finally coalescence. Figure 2 shows the $2 \mathrm{D} \mathrm{Ag}$ clusters formed at three deposition temperatures in the irreversible growth regime. To avoid coarsening of the metastable clusters they have been imaged at the deposition temperature.

Mean-field nucleation theory relates the saturation cluster density $n_{x}$ to the ratio of diffusion $D$ to deposition rate $F$ and to the cluster binding energy $E_{i}$ by the following expression for complete condensation and 2D clusters $[4,50]$

$$
n_{x}=\eta(\theta, i)\left(\frac{D}{F}\right)^{-\chi} \exp \left(\frac{E_{i}}{(i+2) k_{\mathrm{B}} T}\right) \text {, with } \chi=\frac{i}{i+2} .
$$

From (2) it becomes clear that the most direct link between $n_{x}$ and $D$ is obtained at low temperatures where $i=1$. Then the cluster binding energy is by definition $E_{i}=0$, and (2) reduces to

$$
n_{x}=\eta(\theta, 1)(D / F)^{-1 / 3},
$$

with $\eta(\theta, 1)=0.25$ in the coverage range of saturation $[4,18]$. At higher temperature the cluster binding energy $E_{i}$ can for instance be expressed in a pair binding model involving multiples of the energy per bond $E_{\mathrm{b}}$ [46] (for dimers $E_{i}=E_{\mathrm{b}}$, see Fig. 1).

The Arrhenius plot of $n_{x}$ in Fig. 2d shows a roughly linear regime in the range of $10^{5} \leq D / F \leq 10^{9}$, in accordance with (3). The application of this equation to $n_{x}(T)$ data inferred from STM has proven to yield valid numbers for the barrier and attempt frequency of terrace diffusion $[51,52,49]$. It was pointed out that the accuracy of these numbers can be considerably 
a) $T=80 \mathrm{~K}$

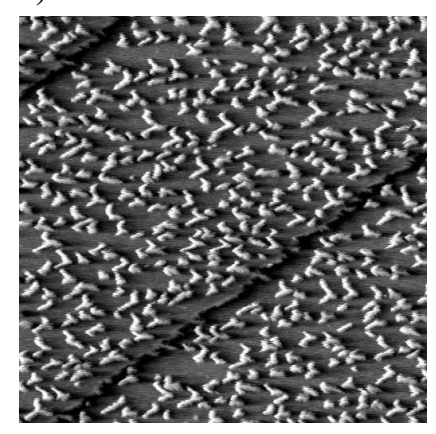

c) $T=110 \mathrm{~K}$

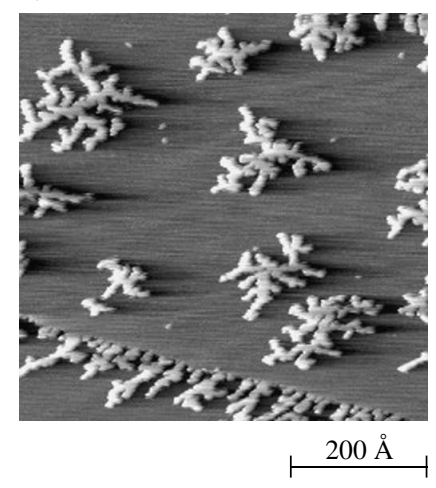

b) $T=95 \mathrm{~K}$
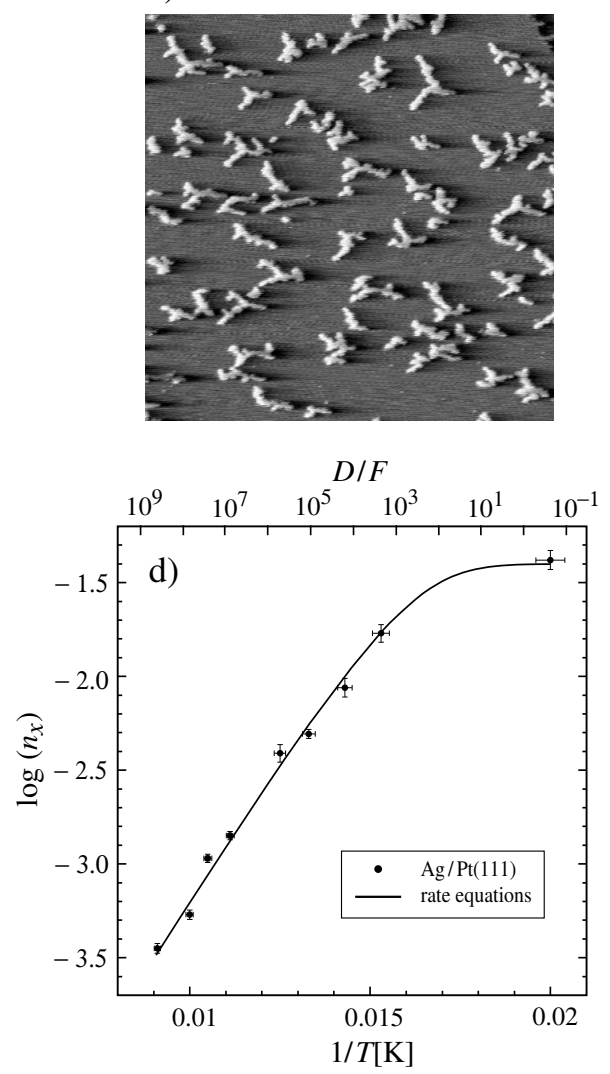

Fig. 2. Variation of saturation island density with temperature for deposition of $\theta=0.12 \mathrm{ML} \mathrm{Ag}$ onto a Pt(111) surface. (a)-(c), Common length scale VT-STM images taken at the respective deposition temperatures. (d) Arrhenius plot of the saturation island densities in the temperature regime where dimers are stable nuclei (for the applied deposition flux of $F=1.1 \times 10^{-3} \mathrm{ML} / \mathrm{s}$ ). Experimental island densities for $\mathrm{Ag} / \mathrm{Pt}(111)$ are compared with results from integrating rate equations from mean-field nucleation theory using self-consistent calculations for capture numbers (solid line) [18]

increased in analyzing cluster densities down to lower temperatures $[18,53]$. There $\left(D / F<10^{5}\right)$, however, (3) no longer holds since monomers are diffusing too slowly to reach each other and create all nuclei during deposition. Therefore monomers are stable nuclei $(i=0)$ in the sense that many of them remain monomers during deposition. However, they continue to diffuse after deposition. This post-deposition mobility gives rise to cluster growth and cluster nucleation in the time between deposition and imaging with STM. It is clearly visible in Fig. $2 \mathrm{~d}$ that this leads to a reduced slope ending with a 
plateau where all islands are created after deposition and thus $n_{x}$ becomes independent from the deposition temperature [49,54]. Post-deposition mobility can be accounted for in rate equations using mean-field nucleation theory. These equations have been integrated using self-consistent solutions to the capture numbers derived by Bales et al. [55]. As a result, the experimental data for $\mathrm{Ag} / \mathrm{Pt}(111)$ could be fitted over a range of almost 10 orders of magnitude in $D / F$ yielding $E_{\mathrm{m}}=168 \pm 5 \mathrm{meV}$ and $\nu_{0}=7 \times 10^{13 \pm 0.3} \mathrm{~s}^{-1}$ [18]. Similar precision was obtained by Bott et al. for $\mathrm{Pt}(111)$ self-diffusion [53].

The experiments studying $n_{x}(T)$ in the irreversible growth regime $(i=1)$ yield a precision for $E_{\mathrm{m}}$ close to that of state-of-the-art FIM studies, which up to now presented our most precise source of information on atomic diffusion barriers. In contrast to FIM, however, the nucleation method is not limited to highly refractive elements and therefore enables the study of monomer diffusion for a wide range of metal and semiconductor systems. In the past, the influence of isotropic strain on diffusion on fcc(111) surfaces was studied [56]. This inspired ab initio calculations revealing that the binding energy of the bridge site becomes less affected by strain than that of the three-fold hollow site, leading to the observed strong effect of strain on $E_{\mathrm{m}}$, which is the difference of both energies [57,41]. Also systems with extremely small diffusion barriers which were formerly inaccessible by experiment, such as $\mathrm{Al} / \mathrm{Al}(111)$ and $\mathrm{Al} / \mathrm{Au}(111)-(\sqrt{3} \times 22)$ could be addressed [10,58,59]. However, the nucleation studies revealing extremely small diffusion barriers $\left(E_{\mathrm{m}}<100 \mathrm{meV}\right)$ systematically yielded prefactors smaller by several orders of magnitude than the universal one discussed above. This may be due to a breakdown of transition state theory, since $E_{\mathrm{m}}$ becomes of the order of $k_{\mathrm{B}} T$ and the adatoms no longer thermally equilibrate between jumps. The low apparent prefactors might equally well indicate the limit of applicability of the nucleation method, i.e., of (3). Since the $E_{\mathrm{m}}$ values are small, the cross-sections of cluster formation get sensitive to small variations in the binding energy of an adatom approaching one of its own. Such variations can be substrate-mediated interactions [60] which in the case of surface sates can be of extremely long range. If these interactions were repulsive over several lattice constants, cluster formation would be delayed with the result of larger cluster densities than expected from (3). Application of this equation would then lead to smaller apparent prefactors.

Apart from this extreme case of exceptionally small $E_{\mathrm{m}}$ values, Eqs. (2) and (3) have been subjected to extensive experimental tests and to tests with Kinetic Monte Carlo (KMC) simulations, all showing that for the general case these equations unambiguously relate $D / F$ to $n_{x}$. Therefore the cluster density at terraces can be tuned for each system to the desired value by choosing an adequate substrate temperature and/or deposition flux. The average cluster size is then adjusted by the coverage.

The cluster size distributions obtained by nucleation on homogeneous substrates all fall onto common curves that depend only on the critical clus- 
ter size $i[61-64,12]$. The scaling law is obtained when the size distributions are plotted as $n_{N} \times\langle N\rangle^{2} / \theta$ vs. $N /\langle N\rangle$, where $N$ and $\langle N\rangle$ are the size and its mean value, and $n_{N}$ is the density of $N$-sized clusters. The shape of the size distributions is Gaussian with a Half Width at Half Maximum (HWHM) of $\sigma \sim 0.55$ in the case of $i=1$ [12]. We will discuss below several methods for significantly narrowing down the size distributions. For the prospect of growing small clusters the low temperature plateau of constant cluster densities shown in Fig. 2d is of interest, since through post-nucleation, exponentially decreasing island size distributions with mostly dimers and trimers are formed.

\section{Aggregation}

The similarity of patterns formed in non-equilibrium growth processes in physics, chemistry and biology is conspicuous, and many attempts have been made to discover common mechanisms underlying their formation $[65,66]$. The most prominent examples are snowflakes. The correlation of their shape with meteorological crystal growth conditions leads to valuable conclusions concerning the meteorology in the upper atmosphere [67]. The manifold growth patterns in nature commonly evolve from non-equilibrium growth at an interface with material transport via diffusion being the rate limiting process. Exactly these conditions govern aggregation of clusters at single crystal surfaces held at low temperatures. Aggregation of submonolayer coverages of metal atoms is often two-dimensional and therefore easier to understand than many more complex growth patterns. Nevertheless, it bears enough complexity that knowledge gained in 2D may be transferred to the understanding of more complicated 3D patterns appearing in nature. This makes low temperature metal aggregation an ideal model system for tracing back mechanisms by which single diffusion events of atoms along a growing interface translate can determine an overall pattern. STM opened access to the structure of monolayer high 2D aggregates formed by atoms at surfaces [68]; and its extension to variable low temperatures enabled the investigation of the kinetics underlying the formation of such patterns down to temperatures where irreversible growth occurs [69-72]. The knowledge gained from microscopic studies in turn provides control over the shape of MBE grown surface-supported clusters. In this section we discuss ramified clusters with fractal dimension, and various compact island shapes formed on isotropic single crystal metal surfaces. For the sake of controlled cluster growth we focus on the link between cluster shape and system parameters and growth conditions.

Let us assume we perform an MBE experiment on an isotropic substrate at low temperature. If adatoms would irreversibly stick to the site where they hit the growing aggregate $(i=1)$, and if edge diffusion were entirely frozen, then so-called Diffusion Limited Aggregation (DLA) -clusters [29,30] would form with monoatomic branches spreading out into random directions. The 
formation of branches is caused by low mobility along the edge leading to the so-called tip- or Saffman-Taylor instability [73]. This instability is due to the fact that protrusions at the edge resulting from statistical fluctuations capture more adatoms from the terrace diffusion field than straight or concave step sections. If these atoms cannot be transported away sufficiently fast, the protrusions grow out as a branch with a width related to the step edge diffusion barrier [74-77]. With increasing length the branch tip becomes exposed to an increased solid angle of aggregating adatoms causing ramification into more branches. The continued ramification leads to the self-similarity of the DLA patterns and a fractal Hausdorff [66] dimension of 1.7, i.e., the cluster area increases as $A=r^{1.7}$, with $r$ being the radius.

a)

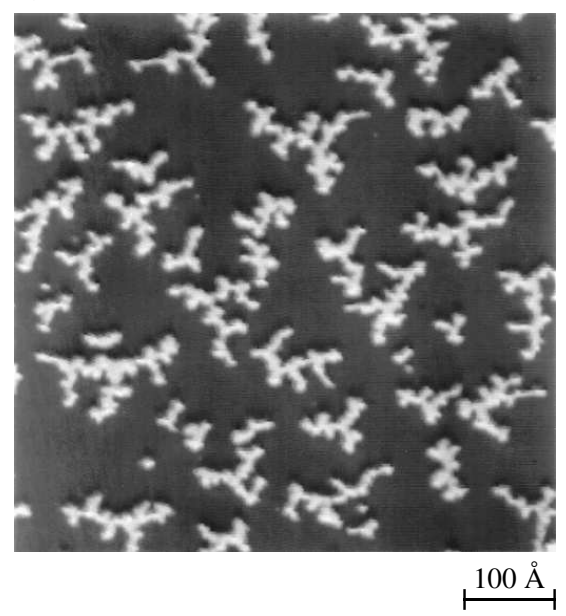

b)

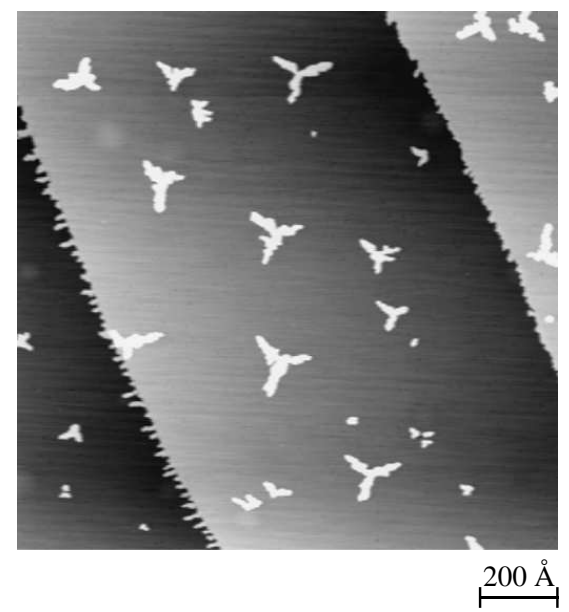

Fig. 3. Dendritic patterns formed for low temperature metal deposition onto hexagonal close-packed substrates. (a) $\operatorname{Pt}(111)$ homoepitaxy at $180 \mathrm{~K}(\theta=$ $0.094 \mathrm{ML}$ ) [78]. (b) Pt heteroepitaxy on $\mathrm{Ru}(0001)$ at $300 \mathrm{~K}(\theta=0.1 \mathrm{ML})$ [79]

However, the classical DLA clusters have so far never been observed in MBE growth on single crystal surfaces and there are strong indications that, if ever observed, DLA growth will be the exception for these systems. Depending on the surface symmetry there are different reasons for the observed lack of DLA clusters. On square lattices there are only one-fold coordinated step sites and therefore edge diffusion has a barrier comparable to that of terrace diffusion [80-82]. As soon as terrace diffusion (the process needed for cluster formation) gets thermally activated, diffusion along the cluster edge is also activates. This generally leads to compact square clusters at any deposition temperature $[83,84,54,85-88]$. An exception is the formation of non-compact islands observed for $\mathrm{Cu} / \mathrm{Ni}(100)$, thought to be due to the strain induced increase of step length [89]. On hexagonally close-packed surfaces, there are cor- 
ner sites with 1-fold lateral coordination and sites at straight $\langle 1 \overline{1} 0\rangle$-oriented edges with 2-fold coordination. Atoms bound to the two-fold edge sites have high $E_{\mathrm{e}}$ values and thus low mobility and this can indeed generate branched clusters (see Figs. 2a-c and 3). However, in contrast to DLA clusters these clusters have trigonal symmetry and their branches are at least 2-3 atoms wide. Nevertheless their fractal dimension is close to the DLA value of 1.7. These islands have been called dendrites by analogy with other patterns in nature revealing preferred growth directions [70]. The three preferred growth directions lead to Y-shapes for small cluster sizes (see Figs. 2a, b and 3b due to the absence of further branching these Y's have a Hausdorff dimension of 1) and to a triangular envelope for larger dendrites (see Figs. 2c and 4c). Note that in the case of $\mathrm{Pt} / \mathrm{Ru}(0001)$ shown in Fig. 3b the substrate has hcp stacking leading to a rotation of the dendrites by $60^{\circ}$ from terrace to terrace.

The reason for the slightly larger than monoatomic branch width in dendrites is connected with the relaxation of 1-fold corner atoms to 2-fold step sites. This relaxation is asymmetric being one of the reasons for the preferred growth directions $[72,78]$. The diffusion asymmetry from corner to edge sites can be inferred from the ball model in Fig. 4a. It shows that there are two different micro-facets A (\{100\}-facet) and $\mathbf{B}$ (\{111\}-facet) formed by the $\langle 1 \overline{1} 0\rangle$-oriented edges of any pseudomorphic cluster placed on a hexagonal substrate. Diffusion from a 1-fold corner-site (C) to an A-step involves an hcp-site at just the right distance from the corner, whereas diffusion to the B-step has either an on top site or the hcp-site very close to the island as transition state. From these geometric considerations one would generally expect that corner to $\mathbf{A}$-step diffusion has a lower barrier than diffusion from a corner to a B-step. This picture is confirmed by EMT $[90,80]$ calculations for a number of metal/metal combinations for which dendritic growth perpendicular to $\mathbf{A}$-steps is observed [10]. Fig. 4b shows the case of $\mathrm{Ag} / \mathrm{Pt}(111)$. Diffusion from a corner site to an $\mathbf{A}$-step has a barrier as low as $E_{\mathrm{m}}$, whereas diffusion to a $\mathbf{B}$-step costs 5 times as much activation energy. KMC simulations (see Fig. 4d) have demonstrated that the asymmetric corner relaxation leads to a preferred population of $\mathbf{A}$-steps over $\mathbf{B}$-steps and thus to the three preferred growth directions. The experimentally observed cluster shapes for $\mathrm{Ag} / \mathrm{Pt}(111)$ (see Fig. 4c) are very well reproduced by these simulations [72].

For some systems such as $\mathrm{Cu}$ and $\mathrm{Pt} / \mathrm{Pt}(111)$, however, EMT calculations show the corner-to-edge diffusion asymmetry to be the other way around [10]. For $\mathrm{Pt} / \mathrm{Pt}(111)$ this result is confirmed by a recent ab initio calculation [91]. $\mathrm{Pt}$ reveals a large tensile stress leading to a strong inward relaxation of the island edge. This can facilitate diffusion towards B-steps as it renders the hcpsite located between corner and $\mathbf{B}$-step more attractive as a transition state; in the absence of strain this site is located too close to the island (see 4a). Despite this inversion of the corner to A- and B-step diffusion asymmetry, $\mathrm{Pt} / \mathrm{Pt}(111)$ shows dendritic growth with the same preferred growth direction as $\mathrm{Ag} / \mathrm{Pt}(111)$ and $\mathrm{Ag} / \mathrm{Ag}(111)$, i.e., perpendicular to $\mathbf{A}$-steps (see Fig. 3a). 
a)

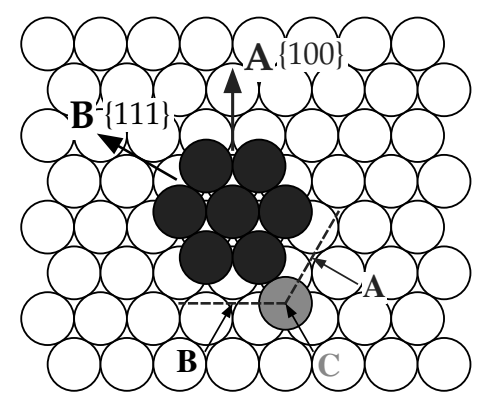

c)

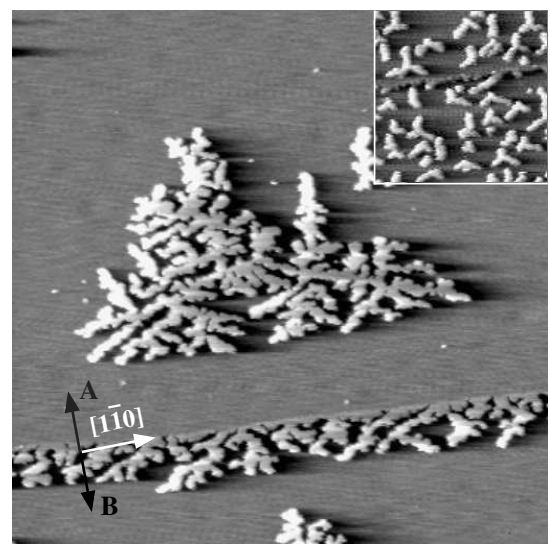

b)

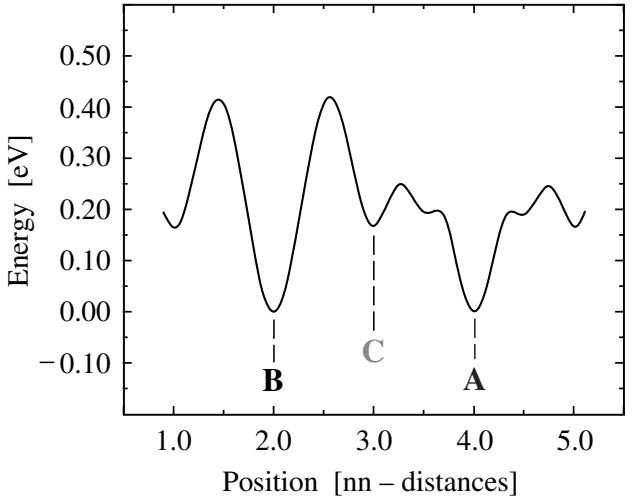

d)

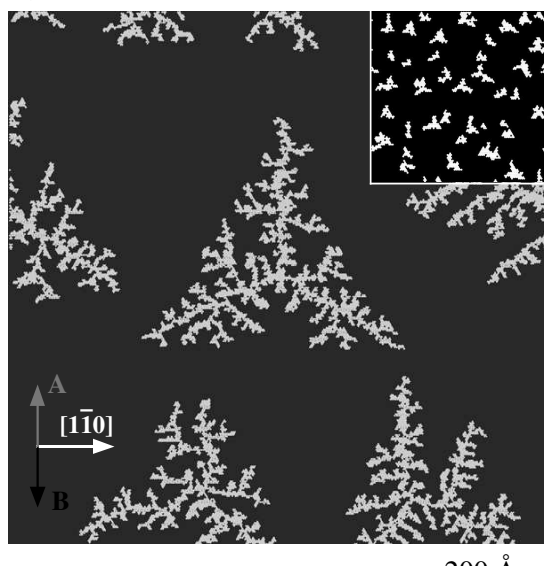

$200 \AA$

Fig. 4. (a) Ball model of a heptamer on a hexagonal lattice revealing the existence of A- and B-steps. (b) The EMT calculation for $\mathrm{Ag} / \mathrm{Pt}(111)$ shows that this geometric difference implies different corner diffusion barriers. (c) Experiment for $\mathrm{Ag} / \mathrm{Pt}(111)$ at $130 \mathrm{~K}$ (main figure) and $80 \mathrm{~K}$ (inset). (d) The KMC simulations identify the difference in corner diffusion as the origin of dendritic growth; they reproduce the dendrites found in experiment (scale bar common to (c) and (d)) [72]

Apart from the aspect of diffusion along the island edge treated so far it was pointed out that the different diffusion paths of atoms from the terrace towards the two kinds of steps give rise to a second asymmetry favoring population of A-steps [72]. Considering the different diffusion paths from second-neighbor fcc sites via hcp sites towards a heptamer one readily finds that there is a strong statistical preference of $3 / 6$ vs. $1 / 6$ for attachment to A- vs. B-steps, whereas corners are visited with a probability of only $2 / 6$ [10]. The attachment asymmetry is more general than corner diffusion 
asymmetry. Both effects have to be considered in conjunction to understand and predict cluster morphology. If corner diffusion is faster towards $\mathbf{B}$-steps, it could counterbalance attachment and random growth may result. For systems with faster diffusion towards A-steps, both effects push towards exclusive population of A-steps resulting in dendritic growth perpendicular to these steps. For $\mathrm{Pt} / \mathrm{Pt}(111)$ there is a slightly smaller barrier for diffusion from corners to B-steps. This anisotropy is too weak to fully counterbalance the preferred attachment to $\mathbf{A}$-steps, leading to dendrites with preferred growth perpendicular to these steps. In agreement with this weak preference, the trigonal symmetry is less pronounced for that system (see Fig. 3a).

The growth of dendrites with trigonal symmetry can be considered as understood. This growth morphology is generally expected for low temperature $(i=1)$ 2D cluster aggregation on hexagonal metal surfaces. Dendritic clusters undergo several mutations upon increasing the temperature and/or reducing the flux, i.e., when going to $i>1$ conditions. For some systems the branches begin to grow into random directions, lifting the trigonal symmetry of the low temperature dendrites [70]; in addition the branches become wider. Despite a considerable effort, the shape transition from dendritic to random isotropic growth patterns $[68,77,92]$ is not yet fully understood. Also a quantitative understanding of the relationship between the degree of edge diffusion and the branch width is presently lacking. We briefly review the present understanding of isotropic fractal clusters. Similar to the dendrites, these patterns reveal the fractal dimension 1.7 of classical DLA aggregate [68].

Examples of this species are shown in Fig. 5. The Au clusters formed at room temperature on $\mathrm{Ru}(0001)$ have branches 60 atoms wide and the $\mathrm{Ag}$ aggregate grown at $220 \mathrm{~K}$ on $\mathrm{Pt}(111)$ has an average branch width of $20 \pm 2$ atoms [77]. The cluster branches spread out and meander into random directions. Formation of partial surface dislocations between fcc- and hcpstacking has been suggested to understand the loss of directional growth [10]. This was motivated by the fact that both systems shown in Fig. 5 reveal considerable misfit possibly leading to partial dislocations where strain is relieved. Each stacking variation from hcp to fcc alters the orientation of A- and B-steps and thus the branches' preferred growth directions. Regular stacking faults could thus explain the observed random isotropic growth. Partial surface dislocations have been observed for $\mathrm{Ag} / \mathrm{Pt}(111)$ upon a critical island size [93], which is however beyond the branch width for which the onset of random growth is observed. It was thus proposed that partials were generated by kinetics, and a KMC model showed that the transition from dendritic to random fractal clusters can be generated that way [10]. A second possible origin for the loss of directional growth is the detachment from 1-fold coordinated corner sites since $i>1$. The arguments of corner diffusion and diffusion paths for attachment to the different step types given above have to be re-examined under reversible growth. This might well lead to the finding 
that frequent detachment of one-fold atoms leads to the observed random growth directions.

a)

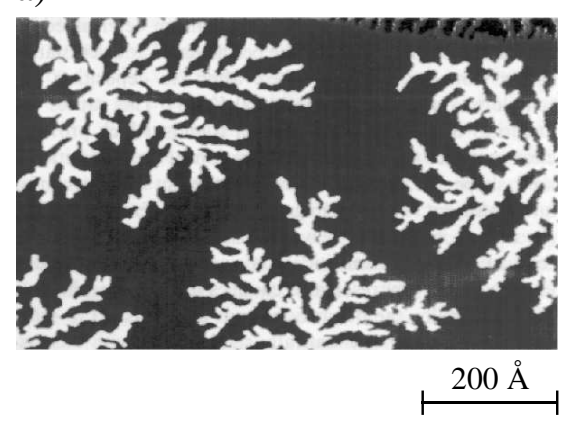

b)

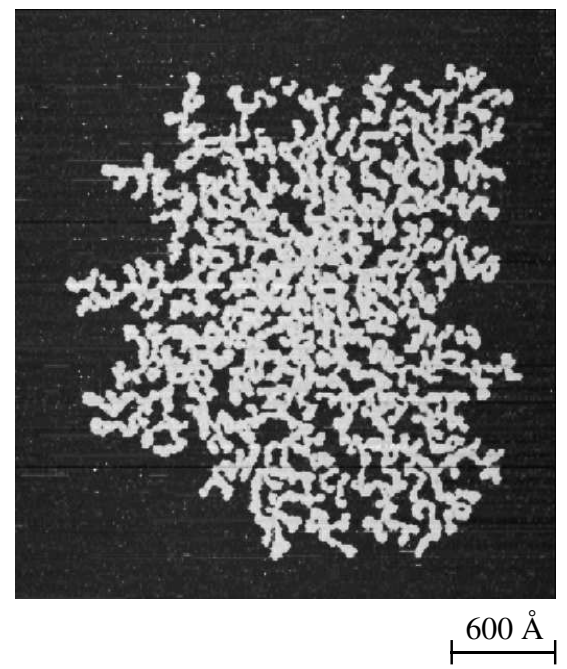

Fig. 5. Examples for random fractals typically following dendrites at elevated temperatures. (a) STM image of $\mathrm{Au}$ islands grown on $\mathrm{Ru}(0001)$ at room temperature $\left(F=3.3 \times 10^{-3} \mathrm{ML} / \mathrm{s}, \theta=0.30 \mathrm{ML}\right)[68]$. The aggregate's branches spread out into random directions. Although the branches are significantly wider than those of the DLA clusters, the aggregate's fractal dimension is the DLA value of 1.72. (b) STM image of a $\mathrm{Ag}$ fractal grown on $\mathrm{Pt}(111)$ at $220 \mathrm{~K}$ similarly showing wide branches that frequently alter their growth direction $\left(F=1.1 \times 10^{-3} \mathrm{ML} / \mathrm{s}, \theta=0.12 \mathrm{M}\right)$ [94]

The branch width was related to edge diffusion, both by analytic models $[88,76,74]$ and through KMC simulations [95]. The basic idea underlying these studies is that the lateral impingement rate $I$ competes with the rate for an adatom to scan the edge of a compact seed particle. The seed particle stays compact until the edges reach a critical width $w$ where both rates become comparable. At that point nucleation of protrusions at the edge can no longer be flattened out and the aggregate becomes unstable upon ramification through the Mullins-Sekerka instability introduced above. This instability argument defines the critical cluster size for ramification and equally the mean branch width taken on by the cluster after ramification. However, the models presented in the literature disagree on the exact dependence of $w$ on $D_{\mathrm{e}} / I$, where $I=F / n_{x}$, and $D_{\mathrm{e}}$ is the $1 \mathrm{D}$ diffusion rate at the edge. Therefore these models give contradicting results for the attempt frequencies and barriers for edge diffusion when experimental data of $w(T)$ (available for $\mathrm{Ag} / \mathrm{Ag}(111)$ and $\mathrm{Ag} / \mathrm{Pt}(111)$ [77]) are analyzed. Realistic models have to incorporate a 
set of parameters where diffusion barriers are attributed to step atoms depending on their coordination and the step orientation. Evidently this leads to the well known problem of models invoking too many parameters to allow a sensible comparison with experiment. This problem is encountered also in recent papers dealing with cluster diffusion, a similarly complex issue [96]. Ways out are either to simplify the model, or to use additional input from calculations to ascribe reasonable values to the barriers. The first attempt has been made by disregarding the difference between $\mathbf{A}$ - and $\mathbf{B}$-steps with a view to extracting an effective $E_{\mathrm{e}}$ value and its attempt frequency for diffusion between 2-fold coordinated sites along straight steps [10]. The result $\left(E_{\mathrm{e}}=370 \mathrm{meV}\right.$ and $\left.\nu_{0}=1 \times 10^{13} \mathrm{~Hz}\right)$ is more realistic than the values obtained from the former analysis [77]. The second attempt will certainly follow in the near future. Ab initio calculations become feasible for larger systems allowing the calculation of various energy barriers for adatom displacement along cluster edges $[97,81,98,91]$, thus generating valuable input parameters for KMC simulations. Random fractal islands with wide branches will certainly be of ongoing interest until we can answer the open question of how they evolve from dendrites and how their branch width is linked to the edge diffusion barrier.

With increasing deposition temperature various compact clusters form on hexagonal substrates. These polygonal clusters are first triangles, whose orientation is set by the trigonal symmetry of the dendrites preceding them at low temperatures. Examples for triangular clusters with preferred orientation are $\mathrm{Co} / \mathrm{Ru}(0001)$ [92,99,100], $\mathrm{Co} / \mathrm{Pt}(111)$ [101], and $\mathrm{Fe} / \mathrm{Au}(111)[102,103]$. The triangles are followed at larger deposition temperature by hexagons, which can mutate back into triangles before reaching the thermodynamic equilibrium shape, by further increasing the deposition temperature or by annealing. The equilibrium shape of a $2 \mathrm{D}$ cluster on a trigonal substrate is a hexagon where, according to the Wulff construction, the different surface free energies of A- and B-steps are reflected in the lengths of these facets [104]. Since interlayer diffusion generally has different barriers for both step types their respective length, or in the case of triangles their orientation, can be decisive for $2 \mathrm{D}$ versus $3 \mathrm{D}$ growth.

The sequence of the various polygonal compact clusters has been reported for $\mathrm{Pt} / \mathrm{Pt}(111)$ [105], and is reproduced in Fig. 6 . At $400 \mathrm{~K}$ triangles bound by A-steps were observed, at $455 \mathrm{~K}$ hexagons, and deposition at $640 \mathrm{~K}$ yielded again triangles, this time bound by $\mathbf{B}$-steps. Finally the clusters attained the quasi-hexagonal equilibrium shape, which was proven to be independent of cluster history (compare Figs. 6d1 and d2).

Inversion of triangle orientation with increasing deposition temperature was a puzzle for theorists $[107-109,81]$ until it recently became solved by a repetition of the experiments under extremely clean evaporation conditions [106]. Taking up the $\mathrm{Pt} / \mathrm{Pt}(111)$ experiments once more was motivated by a discrepancy between results on island orientation and interlayer dif- 
a)

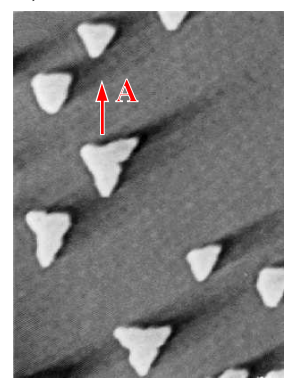

b)

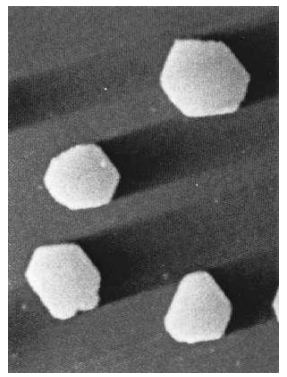

c)

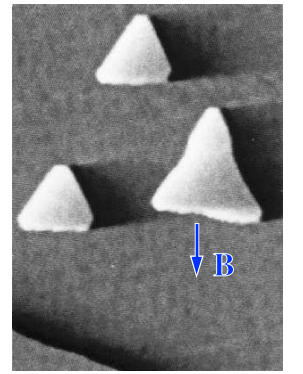

d)

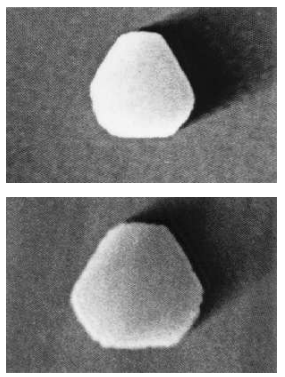

Fig. 6. The formation of various polygonal islands during $\operatorname{Pt}(111)$ homoepitaxy $[105,106]$. The deposition temperatures are as follows. (a) $T=400 \mathrm{~K}$ $\left(\theta=0.08 \mathrm{ML}, 1300 \times 1900 \AA^{2}\right),(\mathbf{b}) T=455 \mathrm{~K}\left(\theta=0.14 \mathrm{ML}, 770 \times 1100 \AA^{2}\right)$, (c) $T=640 \mathrm{~K}\left(\theta=0.15 \mathrm{ML}, 2300 \times 3300 \AA^{2}\right),(\mathbf{d} 1) T=710 \mathrm{~K}(\theta=0.08 \mathrm{ML}$, $\left.1540 \times 1100 \AA^{2}\right),(\mathbf{d} 2)$ deposition at $T=455 \mathrm{~K}$ as in (b) and subsequent annealing to $710 \mathrm{~K}$ for $1 \mathrm{~min}\left(\theta=0.08 \mathrm{ML}, 630 \times 900 \AA^{2}\right)$. Common deposition flux $F=1 \times 10^{-2} \mathrm{ML} / \mathrm{s}$

fusion, and ab initio calculations [36]. The experiments led to the important conclusion that minute amounts of impurities (CO) were responsible for inversion of triangle orientation. Depositing at extremely low CO partial pressure $\left(p_{\mathrm{CO}} \leq 5 \times 10^{-12}\right.$ mbar, see Fig. 7$)$ the island shape evolves from dendrites via fractals with large branches to triangles only bound by B-steps; the triangles increase in size with increasing temperature but they keep their orientation [106].

Before we go into the details specific to the system $\mathrm{Pt} / \mathrm{Pt}(111)$, let us discuss the mechanism generally responsible for triangular cluster shapes on trigonal substrates. In the kinetic regime, the cluster shape is determined by the growth rate perpendicular to $\mathbf{A}$ and $\mathbf{B}$ steps, i.e., by the rate with which adatoms accumulate at both steps. The slowly growing facets prevail in the final crystal shape whereas the faster ones disappear during growth, as is generally the case in crystal growth. There are two temperature regimes, in each of which the step progression rate is determined by different atomic processes. At temperatures where diffusion around corners is frozen, and thus material exchange between both step types inhibited, the diffusion rates along the two edges will determine the cluster shape. The step type with fast edge diffusion grows smoothly with few kinks, whereas at the other step edge diffusion is slow and growth involves many kinks. Hence, the first step propagates only slowly whereas the latter progresses rapidly [105,109]. We note, however, that this first case can be artificial since barriers for corner crossing are often quite close to those for edge diffusion $[98,91]$. Therefore the temperature regime where corner crossing is frozen but edge diffusion active is small, if it exists at all. Much more important is the case where corner crossing becomes activated. The diffusion bias around the corners between $\mathbf{A}$ and $\mathbf{B}$-steps, and 
the progression of the respective steps, is given by the difference in their adatom binding energies, $\Delta E_{\mathbf{A}-\mathbf{B}}=E_{\mathbf{A} \longrightarrow \mathrm{c}}-E_{\mathrm{c} \longrightarrow \mathbf{A}}-E_{\mathrm{c} \longrightarrow \mathbf{B}}+E_{\mathbf{B} \longrightarrow \mathrm{c}}$ (c denotes the one-fold corner site) [110]. Thus the shape of compact islands, in the kinetic regime, depends on a small difference of large activation energies. If both edges bind adatoms equally well compact irregular spherical islands form, if there is a small energy difference triangles form, their orientation being given by the sign of the binding energy difference.
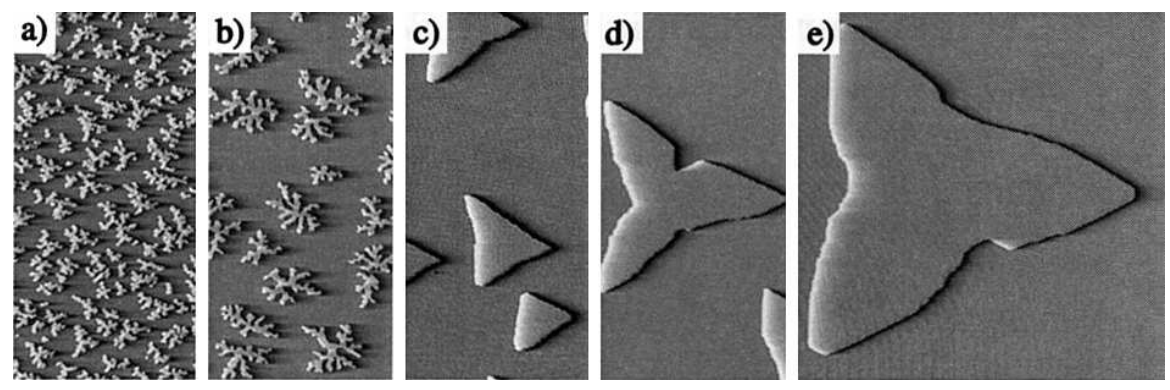

Fig. 7. Island morphology for $0.17 \mathrm{ML} \mathrm{Pt} / \mathrm{Pt}(111)$ under extremely clean evaporation conditions (during evaporation $p_{\text {tot }}<2.0 \times 10^{-11} \mathrm{mbar}$ ) [111]. The deposition temperatures are as follows. (a) $T=200 \mathrm{~K}$, (b) $T=300 \mathrm{~K}$, (c) $T=400 \mathrm{~K}$, (d) $T=500 \mathrm{~K},(\mathbf{e}) T=600 \mathrm{~K}\left(630 \times 900 \AA^{2}\right)$, image sizes $(\mathbf{a}-\mathbf{d}) 670 \times 1340 \AA^{2},(\mathbf{e})$ $1340 \times 1340 \AA^{2}$

Accordingly, the triangles with B-steps shown in Figs. 7c-e are due to better binding to A-steps. Note that this is in contradiction with recent results from theory [91]. The binding energy difference obtained in these calculations is weak, but it favors population of $\mathbf{B}$-steps, and this is not in agreement with their prevalence. Triangles with the inverse orientation, however, are due to minor amounts of $\mathrm{CO}$ present during deposition. The effect of CO adsorption on the energetics and kinetics of edge diffusion has not yet been explored theoretically.

The example of $\operatorname{Pt}(111)$ homoepitaxy shows that the island shape is a sensitive indicator for small energy differences which can therefore be rather useful to test ab initio calculations. It is clear that the binding energy difference between the two step types is a thermodynamic argument, however, it also determines the diffusion bias around corners in the kinetic regime. In thermodynamic equilibrium the step formation energy comes into play. The total step length is then reduced by the formation of quasi hexagons. The example of $\mathrm{Pt}(111)$ homoepitaxy also shows that the effect of defects does not belong to the past even in careful UHV experiments. Chemically influenced diffusion is now being systematically addressed for a number of metal/metal systems $[112,113]$. 


\section{Coarsening}

Coarsening by Ostwald ripening is a means of preparing compact surface supported 2D clusters with narrow size distributions and average sizes $\langle N\rangle$ adjustable from 3 to $10^{3}$ atoms [69]. This is of particular importance, since currently much experimental and theoretical effort focuses on exploring the evolution of chemical and physical properties of small agglomerations of matter as a function of the number of atoms they contain [114].

a) $50 \mathrm{~K}$

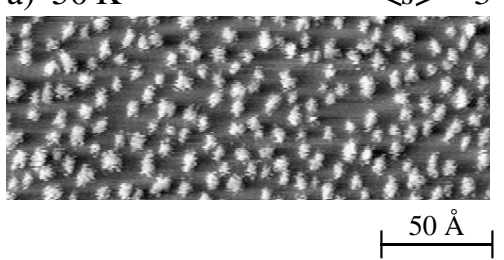

b) $110 \mathrm{~K}$

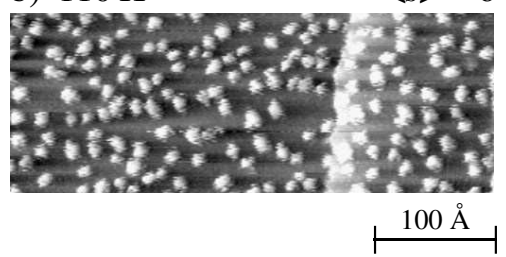

d) $230 \mathrm{~K}$

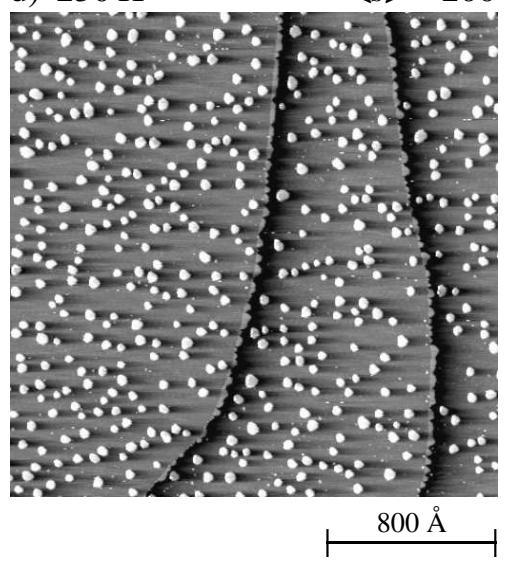

c) $140 \mathrm{~K}$

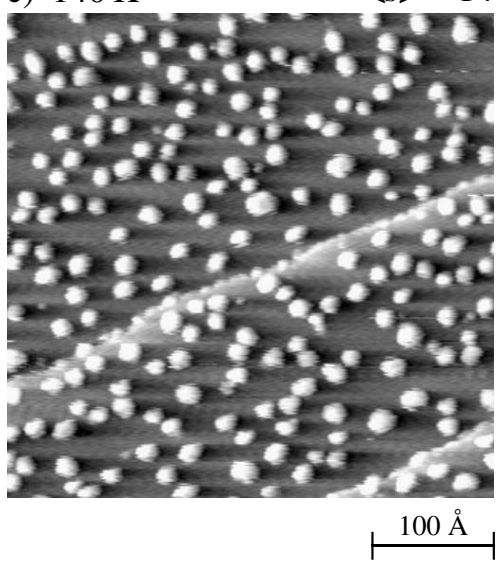

e) $280 \mathrm{~K}$

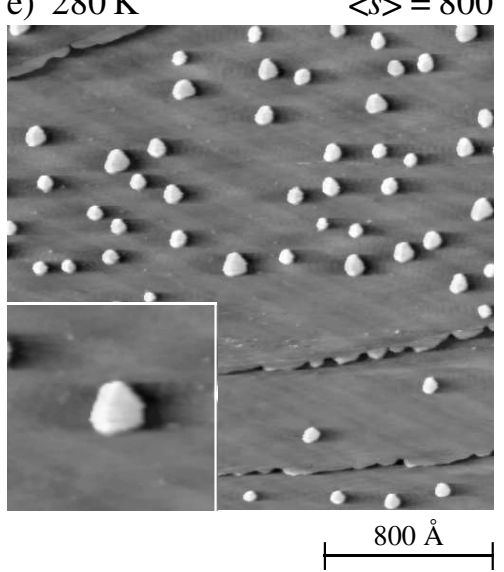

Fig. 8. STM images showing Ostwald ripening in two dimensions as a way to create compact $2 \mathrm{D}$ islands with their size $\langle N\rangle$ being well defined by the annealing temperature [69]. The starting population of mostly dimers and trimers was produced via deposition of $0.1 \mathrm{ML} \mathrm{Ag}$ onto $\mathrm{Pt}(111)$ at $50 \mathrm{~K}$ 
Ostwald ripening [115-117] is caused by a more rapid dissociation of smaller islands in favor of larger ones. Through the availability of surface microscopy at the atomic level this phenomenon has received considerable attention in two dimensions $[69,118,119]$.

The starting point for the preparation method is a large density of small clusters, mostly dimers, prepared by deposition of $\sim 0.1 \mathrm{ML}$ at a temperature chosen such that there is little mobility in the time of deposition $\left(D / F<10^{3}\right)$. In this post-nucleation regime (see Sect. 3) monomers diffuse towards each other mostly after deposition leading to a mean cluster size of $\langle N\rangle \sim 3$ atoms. The same result is obtained when depositing at temperatures where diffusion is frozen and subsequently gently annealing the surface to activate diffusion [54,18]. After preparation of the small clusters, their densities and thus their average sizes (since $\theta$ is a known constant) are monitored by STM as a function of annealing temperature. The Ag clusters on Pt(111) shown in Fig. 8 are compact spherical until they adopt a quasi-hexagonal shape with different lengths of the $\mathbf{A}$ - and $\mathbf{B}$-facets. This shape can be considered as the equilibrium shape of a $2 \mathrm{D}$ cluster for that system since further annealing leads to island decay. This is believed to be promoted by the compressive strain inherent in the clusters $[120,121]$.

a)

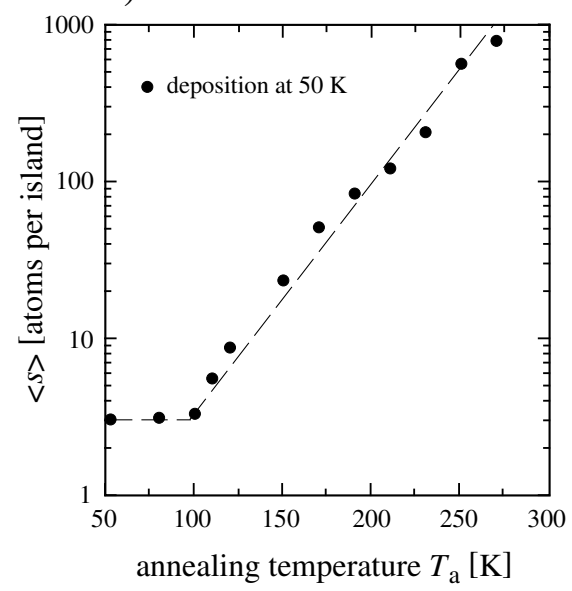

b)

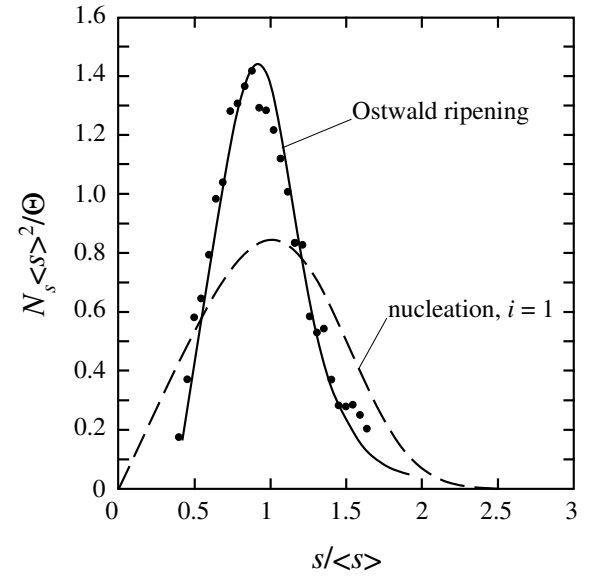

Fig. 9. Investigation of $2 \mathrm{D}$ Ostwald ripening for $\mathrm{Ag} / \mathrm{Pt}(111)$ by means of STM [10]. (a) The mean island size $\langle N\rangle$ stays constant until it exhibits an exponential increase due to Ostwald ripening for annealing temperatures $T_{\mathrm{a}}>100 \mathrm{~K}$. (b) Scaled island size distributions for Ostwald ripening (data from Fig. 8, solid line serves as guide to the eye) are significantly more narrow as compared to nucleation (dashed line theoretical $i=1$ scaling curve [12]) 
The evolution of the mean island size while annealing shows a plateau followed by an exponential increase characteristic of Ostwald ripening (see Fig. 9a). The constant regime implies that the most fragile objects in the population, namely the dimers (and on square lattices also the trimers), neither dissociate nor diffuse, since both would lead to coarsening. The temperature threshold for the onset of 2D Ostwald ripening thus defines the $i=1$ regime and yields the dimer dissociation barrier, which contains the dimer bond energy $E_{\mathrm{b}}$ via $E_{\mathrm{diss}} \sim E_{\mathrm{m}}+E_{\mathrm{b}}\left(E_{\mathrm{b}}=150 \pm 20 \mathrm{meV}\right.$ has been inferred in this way for $\mathrm{Ag} / \mathrm{Pt}(111)$ [18]).

As an advantage to island nucleation, the size distributions obtained from Ostwald ripening, starting from the exponentially decreasing size distributions of post-nucleation [54], are significantly sharper. This becomes evident from inspection of the STM images in Fig. 8 and from the size distributions shown in Fig. 9b. The half width at half maximum decreases from $\sigma=0.55$ for regular $i=1$ nucleation to $\sigma=0.3$ for coarsening. Similar to nucleation, the island size distributions obtained from Ostwald ripening at various temperatures become congruent when scaled the same way as for nucleation.

\section{Anisotropic Surfaces}

Anisotropic substrates show directional dependence of adatom diffusion rates and/or of lateral sticking coefficients of adatoms to clusters. Both effects lead to the creation of elongated clusters, in the extreme case of one-dimensional chains of atoms (see Figs. 10a and 11a). The 1D structures can be considered as quantum wires, the physical properties of which are of considerable scientific interest. Their fabrication with high abundance by MBE growth is thus highly appreciated.

There are various ways to grow quasi-1D clusters at surfaces. One is step decoration [122-124] that has advanced as far as the controlled row-by-row growth at steps of vicinal surfaces [125-127]. We focus our present discussion, however, on clusters formed amid substrate terraces. The desired 1D or elongated structures imply the use of anisotropic substrates. Candidates for metal substrates with diffusion anisotropy are the hex-reconstructed fcc(100) surfaces of Au, Pt and Ir [128,129]. The clusters formed on these surfaces are rectangles elongated along the direction of fast diffusion [130-133]. However, pure 1D structures do not form since sticking to clusters is isotropic on these surfaces. In addition, cluster formation on the hex-reconstructed surfaces is associated with lifting the underlying reconstruction and this makes an understanding on the atomic level difficult. Substrates that permit the growth of 1D strings of atoms, and where cluster formation is easier to understand, are the $(1 \times 2)$ reconstructed and unreconstructed fcc $(110)$ surfaces. We will concentrate on these surfaces where anisotropic diffusion and anisotropic lateral bonding of adatoms in conjunction lead to real 1D clusters. 
a)

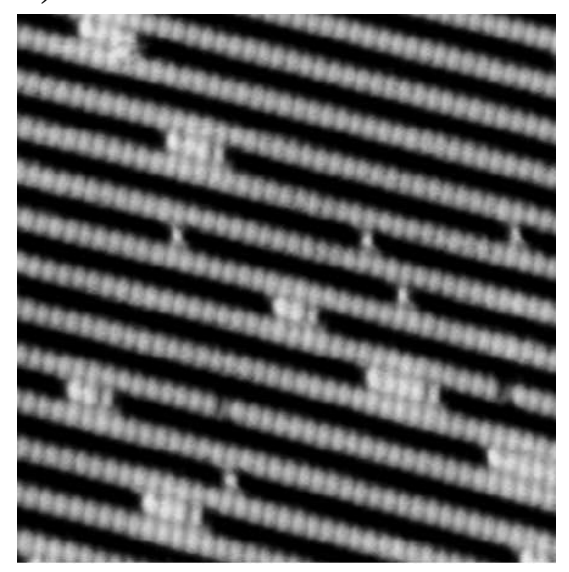

b)

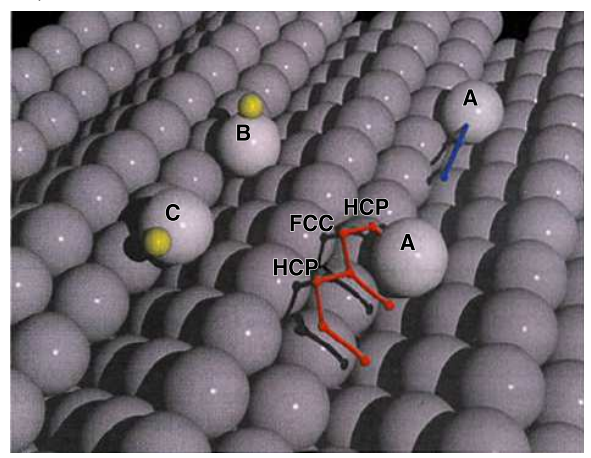

c)

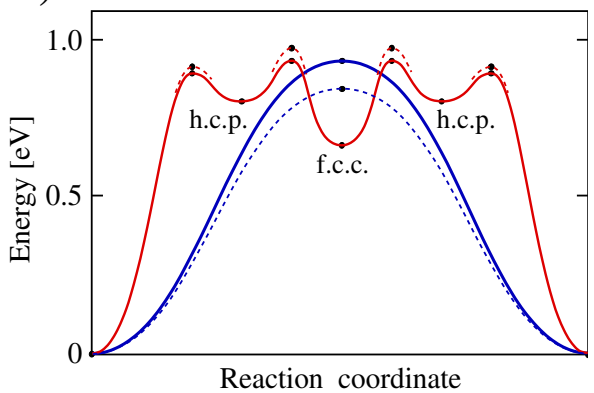

Fig. 10. (a) STM image showing the $(1 \times 2)$-reconstructed $\operatorname{Pt}(110)$ surface after a submonolayer amount of $\mathrm{Pt}$ has been deposited at $313 \mathrm{~K}$ (image size $97 \times 102 \AA$ ) [134]. (b) Model of the $(1 \times 2)$ reconstruction showing two possible diffusion paths for a $\mathrm{Pt}$ adatom $(\mathbf{A})$ along the troughs. The first is direct and the second indirect over fcc and hcp sites on the $\{111\}$-oriented ridge. (c) Ab initio-calculation of the total energy along the reaction coordinate for both diffusion processes ( full lines). Both diffusion paths are degenerate in activation energy. In the presence of atomic $\mathrm{H}$ this degeneracy is lifted and the direct diffusion path has a considerably lowered barrier (dashed lines) [112]

The clean $\mathrm{Pt}(110)$ surface exhibits a $(1 \times 2)$ reconstruction where every other close-packed atomic row is missing, leading to troughs bound by the energetically favored $\{111\}$-facets (see Fig. 10b). Diffusion of Pt adatoms on this surface is one-dimensional and occurs by a certain number $(\sim 10 \%)$ of double jump events, i.e., jumps to second neighbor sites, as revealed from an analysis of time-lapsed STM images [134] such as the one shown in Fig. 10a. An adatom (labelled $\mathbf{A}$ in Fig. 10b) has two diffusion paths to move along the trough, a direct one along the bottom of the trough, and an indirect one where the atom walks 'up' on the $\{111\}$-facet. There it passes an hcp, an fcc and another hcp site before it goes down again to the bottom of the trough. Calculations with DFT (Fig. 10c) showed that both reaction paths 
have equal activation energies [112]. The existence of the two diffusion paths has also been found by molecular dynamics (MD) simulations for self-diffusion on the $(1 \times 2)$-reconstructed $\mathrm{Au}(110)$ surface. These simulations show that the indirect path is the cause for the long jumps [135]. DFT calculations show that the degeneracy of paths is lifted by adsorption of atomic hydrogen (see dashed lines in Fig. 10c). Further more it is found that the activation energy is lowered as compared to the clean case for diffusion of the $\mathrm{Pt}-\mathrm{H}$ complex (B and C in Fig. 10b) along the bottom of the troughs. In accordance with the smaller activation energy STM revealed significantly enhanced diffusion rates for the $\mathrm{Pt}-\mathrm{H}$ complexes showing up as brighter $\mathrm{Pt}$ adatoms in constant current topographs [112].

Apart from the lessons regarding the complex pathways of 1D surface diffusion and its sensitivity to adsorbates, the example of $\mathrm{Pt} / \mathrm{Pt}(110)-(1 \times 2)$ also illustrates the effect of anisotropic sticking on the cluster shape. The clusters in Fig. 10a are 1D strings a few atoms in length. The 1D shape is caused by the fact that diffusion along troughs neighboring chains is unperturbed; the distance between two troughs is large and interaction across the ridge is weak. In contrast to the negligible lateral sticking coefficient the binding energy of an atom at the end of an atomic string is large. Small 1D clusters have also been observed for $\mathrm{Au}, \mathrm{Ni}$, and $\mathrm{Cu}$ deposition on the $(1 \times 2)$-reconstructed $\mathrm{Au}(110)$ surface $[17,16,136]$. We note that above certain temperatures these systems involve exchange diffusion leading in heteroepitaxial cases to alloyed islands and ridges.

The anisotropies of diffusion and sticking are much weaker on the unreconstructed fcc(110) surfaces since there the troughs are closer and the ridges between them are less protruding. Nevertheless, it was on the unreconstructed surface that 1D metal clusters where observed for the first time $[69,137]$. The STM image reproduced in Fig. 11a shows chains of $\mathrm{Cu}$ atoms aligned along the troughs of the $\mathrm{Pd}(110)$ surface. The formation of monoatomic $\mathrm{Cu}$ wires up to $1000 \AA$ long was reported for that system, corresponding to aspect ratios as large as $A \sim 300$ [69]. With increasing deposition temperature, to values above room temperature, clusters become compact while remaining elongated along the $\langle 1 \overline{1} 0\rangle$-direction. Atomic chains were also observed for $\mathrm{Pd}$ [138] and $\mathrm{Fe}$ [139] deposition onto $\operatorname{Pd}(110)$.

The mechanism underlying cluster growth for $\mathrm{Cu} / \mathrm{Pd}(110)$ and in general on unreconstructed fcc(110) surfaces has been the subject of various models. A KMC model (see Fig. 11c) accounting for the fcc(110) symmetry involves the following assumptions. Diffusion within a trough adjacent to a chain is unperturbed $\left(E_{\mathrm{h}, y}=E_{\mathrm{e}, y}=0.3 \mathrm{eV}\right)$ whereas adatoms are less mobile along the short cluster edge $\left(E_{\mathrm{e}, x}=0.65 \mathrm{eV}\right)$; cross channel diffusion was allowed $\left(E_{\mathrm{h}, x}=0.45 \mathrm{eV}\right)$ and a net mass transport along the island perimeter towards the short island end was incorporated (corner rounding $E_{\mathrm{c}, y}=0.3 \mathrm{eV}$, whereas $\left.E_{\mathrm{c}, x}=0.65 \mathrm{eV}\right)$. The model reproduced well the temperature dependence of average experimental quantities such as cluster aspect ratio (see 
a) $T=300 \mathrm{~K}$

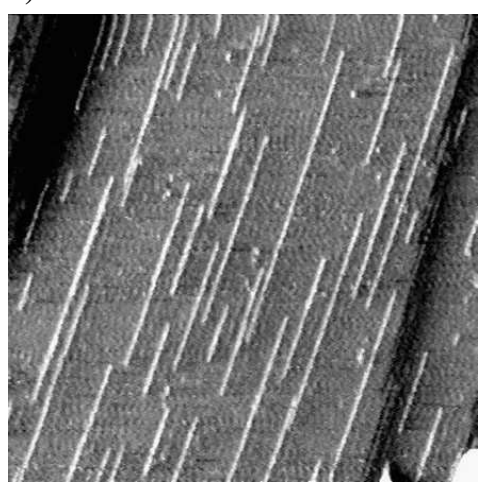

c)

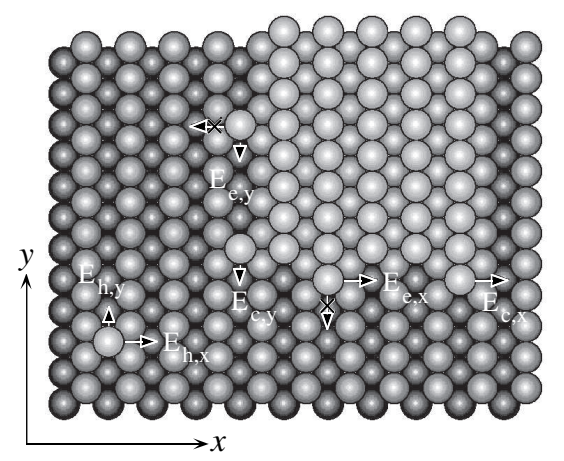

b) $T=320 \mathrm{~K}$

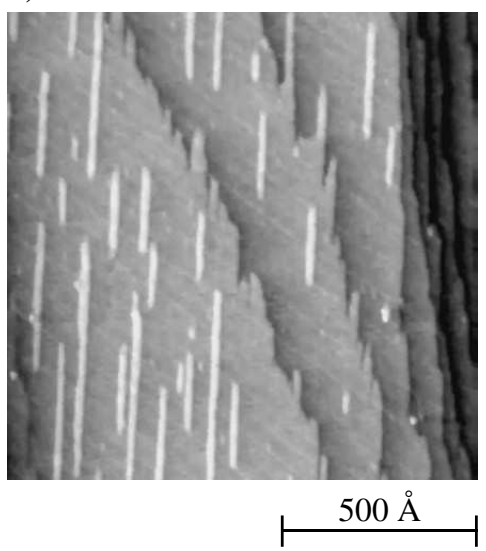

d)

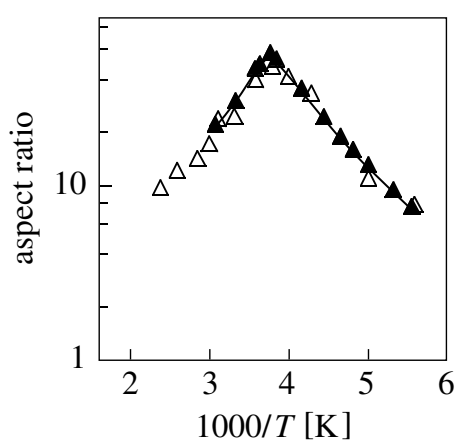

Fig. 11. (a) STM image of monoatomic $\mathrm{Cu}$ chains on $\mathrm{Pd}(110)$ grown at room temperature $(\theta=0.07 \mathrm{ML})$. (b) Rectangular clusters form upon deposition at $320 \mathrm{~K}(\theta=0.1 \mathrm{ML}$, common scale bar for (a) and (b)) [137]. (c) Anisotropic edge diffusion and sticking are the dominant mechanisms giving rise to elongated islands on fcc(110) surfaces. The ball model shows the processes considered in the KMC simulation (for simulation parameters see text). (d) Simulated (filled symbols) vs. experimental (open symbols) cluster aspect ratios [140]

Fig. 11d) and density [140]. According to the simulations, the 1D cluster regime is extended to temperatures far beyond the 1D diffusion regime, leading to the large chain lengths. This is due to the corner rounding process, i.e., to adatoms diffusing along the chains and then attaching via cross channel diffusion to chain ends. The 2D cluster shape at higher temperatures is caused by transport from short to long island edges competing with the opposite one. With a second model it was argued that the mechanism leading to the morphology transition from 1D chains to $2 \mathrm{D}$ clusters is not direct diffusion 
around the corner but rather detachment, terrace diffusion and reattachment to the cluster $[141,142]$.

The essential point to be learned from the KMC simulations is that anisotropic sticking (or similarly anisotropic adatom diffusion around the cluster perimeter) is much more important for the cluster shape than diffusion anisotropy. This fact is illustrated by $\mathrm{Si} / \mathrm{Si}(100)$ where anisotropic sticking and diffusion anisotropy are turned by $90^{\circ}$ to each other. Since the role of sticking overwhelms that of diffusion, clusters are elongated along the slow diffusion direction [143]. Nevertheless diffusion anisotropy is essential for the understanding of island density scaling with flux and temperature. On the $(1 \times 2)$-reconstructed fcc $(110)$ surfaces diffusion is strictly $1 \mathrm{D}$. Thus there is no possibility of net adatom flux around the cluster perimeter explaining the fact that 1D clusters on these surfaces are generally much shorter than on unreconstructed ones.

\section{Growth of Cluster Arrays}

The strain energy present in heteroepitaxial systems and the surface stress characterizing clean surfaces can give rise to the formation of weakly incommensurate layers, respectively surface reconstructions. On fcc(111) surfaces these layers have surface partial dislocations marking transitions between fcc and hcp-stacking domains. These dislocations (or domain walls) have longrange mutual repulsive interactions, and this has two general implications. Firstly, there is a well defined density of dislocations due to the compromise between optimum strain relief achieved through introduction of dislocations and their mutual repulsion. Secondly, the dislocations order into regular patterns. Examples are the herringbone reconstruction of $\mathrm{Au}(111)$ (see Fig. 12a), or dislocation networks (for a general account of strain relief on fcc(111) surfaces see [144-146]).

There is clear experimental evidence that for many epitaxial systems the surface partial dislocations represent strongly repulsive line defects for diffusing adatoms $[56,147,58]$. Their influence on nucleation can go as far as to drive the most perfect layer-by-layer growth yet observed [148]. The two ingredients, ordering of dislocations into periodic patterns and strong influence of dislocations on adatom diffusion, can be employed to grow periodic arrays of almost monodispersed islands [149]. In this section we will discuss results obtained for nucleation on such patterned substrates.

Our first example is Ni nucleation at the 'elbows' of the $\mathrm{Au}(111)(\sqrt{3} \times 22)$ reconstruction. Fig. 12a shows the clean $\mathrm{Au}(111)$ surface with the $\langle 11 \overline{2}\rangle$ oriented partials appearing bright since they involve bridge sites. Strain relief is unidirectional in the $(\sqrt{3} \times 22)$ unit cell. To achieve overall isotropic strain relief a well ordered mesoscopic pattern of two domains with alternating orientation of $\pm 120^{\circ}$ evolves on large terraces. The Ni clusters formed at room temperature deposition are all lined up along the elbows of this so-called 
a)

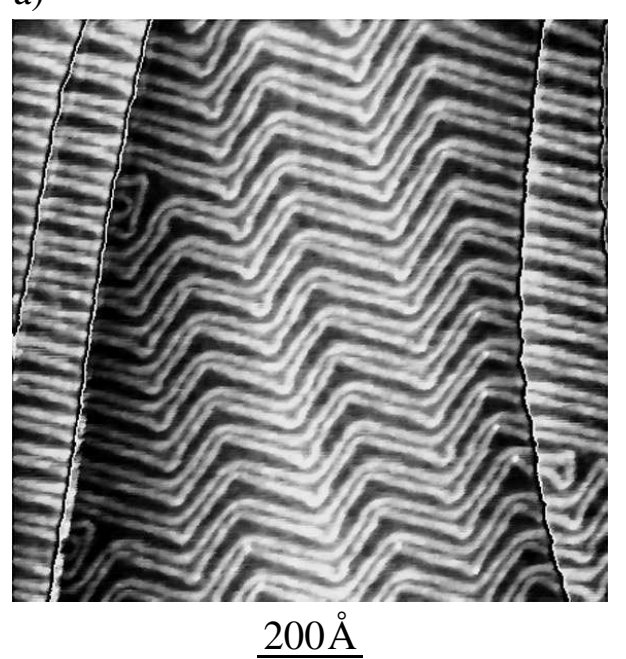

b)

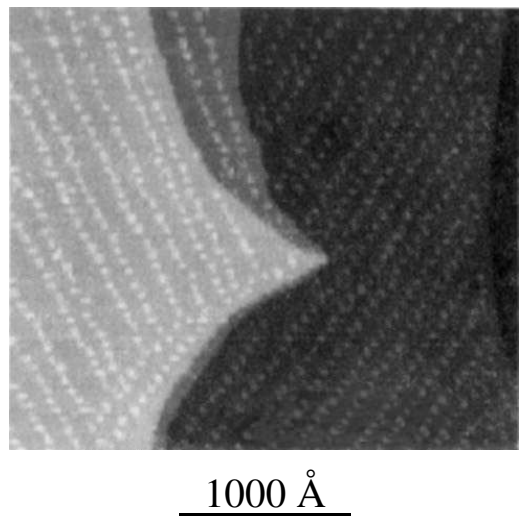

c)

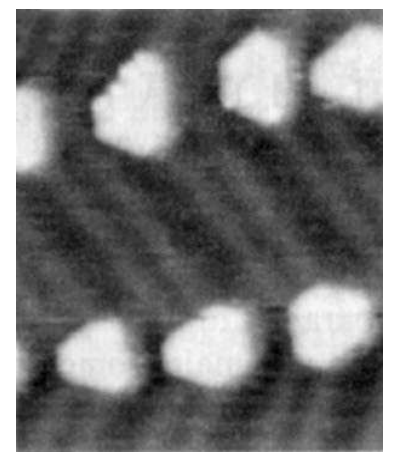

$200 \AA$ d)

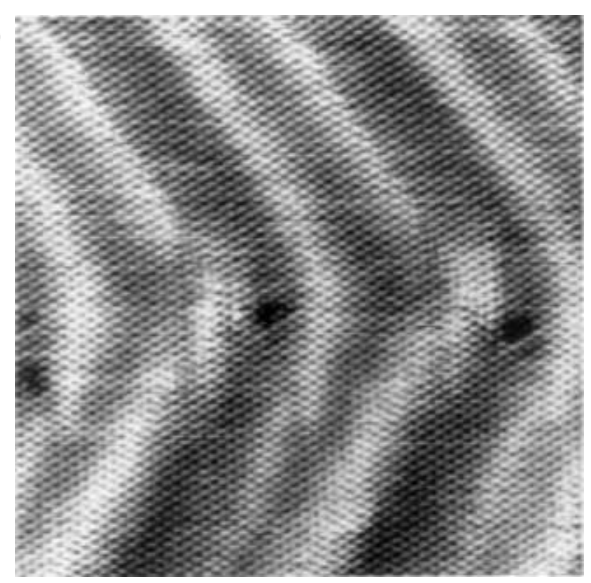

Fig. 12. (a) STM image of the herringbone pattern characterizing the mesoscopic order of the $\mathrm{Au}(111)-(\sqrt{3} \times 22)$-reconstruction [150]. Partial surface dislocations are imaged $0.20 \AA$ higher than fcc areas and thus appear bright. Ni nucleation on that surface at room temperature takes place exclusively at the elbows of the reconstruction (b) $(\theta=0.11 \mathrm{ML})$ leading to monolayer high $\mathrm{Ni}$ islands aligned in rows along the $\langle 11 \overline{2}\rangle$-directions $(\mathbf{c})(\theta=0.14 \mathrm{ML})[151]$. This preferential nucleation at elbows is due to exchange of $\mathrm{Ni}$ into $\mathrm{Au}$ at these sites [152]. (d) The formation of adislands can be suppressed by performing adsorption at $350 \mathrm{~K}$ giving access to the embedded Ni islands; they are comprised of 4 to 5 atoms each $(\theta=0.002 \mathrm{ML})$ 
herringbone reconstruction pattern (Figs. $12 \mathrm{~b}$ and c). Note that the regular spacing is accompanied by narrow cluster size distributions. The ordered nucleation was long believed to be caused by the influence of dislocations on diffusion. They were thought to generate attractive potential dips at the elbows [151]. However, the origin of ordered nucleation was revealed by Meyer et al. to be site selective exchange of $\mathrm{Ni}$ atoms with $\mathrm{Au}$ surface atoms [152]. The exchange is localized at the elbows since there a close-packed atomic row terminates, giving rise to $\mathrm{Au}$ atoms with reduced lateral coordination which are especially susceptible to exchange processes. The embedded $\mathrm{Ni}$ clusters each comprising only $4-5$ atoms are clearly detected as depressions in Fig. 12d. The site selective exchange is followed by preferential nucleation of $\mathrm{Ni}$ adislands on top of substitutional $\mathrm{Ni}$ islands; the adislands appear bright in Figs. 12b and c.

The ordering mechanism present in the $\mathrm{Ni} / \mathrm{Au}(111)$ system is presumably also active for Fe [102,103], Co [153,154] and Rh [155] showing clusters lined up at elbows, too. The argument given by Meyer et al. was that these elements have a larger surface free energy and heat of sublimation than $\mathrm{Au}$ [152]. Accordingly, for elements with lower values of these quantities such as $\mathrm{Ag}$ [156] and $\mathrm{Al}$ [58] the ordering is absent. However, predicting instability towards exchange solely on the basis of bulk quantities does not always work; $\mathrm{Al} / \mathrm{Au}(111)$ shows exchange at $T>245 \mathrm{~K}[157,158]$. Nevertheless, the picture is consistent that site selective exchange is generally responsible for ordered nucleation on the elbows of the reconstructed $\mathrm{Au}(111)$ surface. With that in mind it is clear that ordering is specific to that surface and to the elements of the periodic table exhibiting exchange on it.

A more general approach relying on pure adatom diffusion on networks of dislocations has been suggested [149]. Dislocations confine adatoms by their repelling them into the unit cell in which they were deposited leading to the nucleation of exactly one cluster per unit cell. The partial dislocations are a smooth stacking transition extended over many atoms, as is the repulsion of adatoms away from the dislocations. In addition, fcc- and hcp-domains often have different adatom binding energies. Both effects cause clusters to form on a well defined site within the unit cell, thereby congruently transferring the order of the template surface to the cluster array.

Figure 13 illustrates an example where an array of 2D Ag clusters formed on the dislocation network of $2 \mathrm{ML} \mathrm{Ag}$ on the Pt(111) surface. The misfit between $\mathrm{Ag}$ and $\mathrm{Pt}$ leads to a $(25 \times 25)$-network with surface partials representing 'soft' walls, with lower surface atom density, were the compressive strain is relieved [144] (Fig. 13a). Ag nucleation on top of this network reveals a transition between two rate limiting diffusion processes with an intermediate temperature regime where exactly one island forms per unit cell (see Figs. 13b and d). Note that all of the clusters nucleate on the distorted hexagon of the unit cell implying preferential binding to these fcc-stacking areas, in agreement with theory [159]. The Arrhenius slopes (Fig. 13d) below 
a)

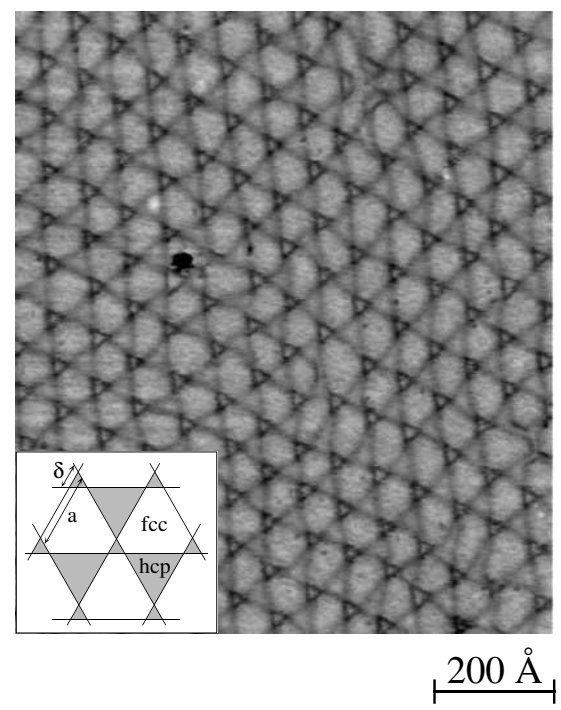

c)

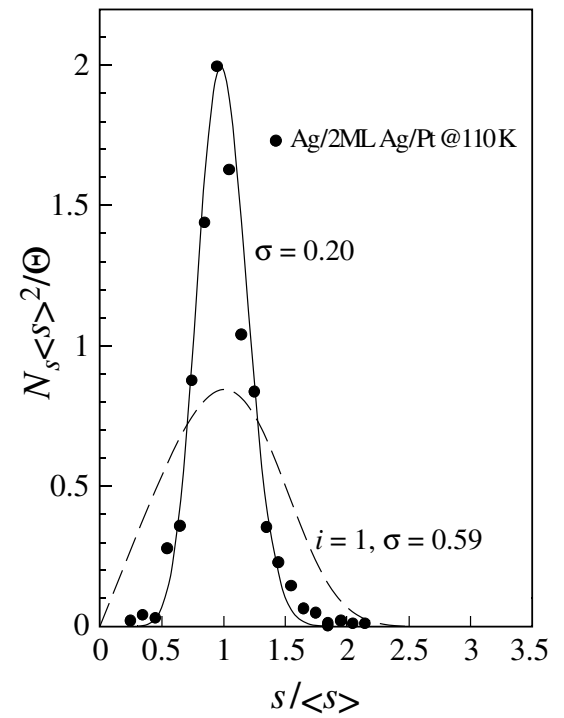

b)

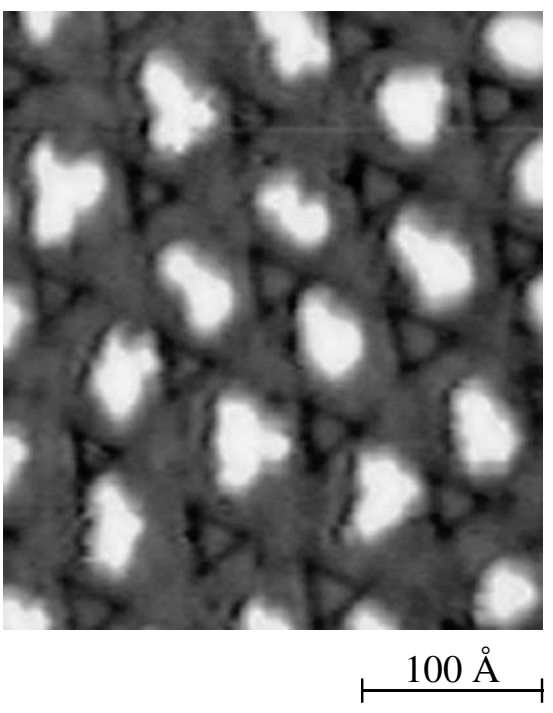

d)

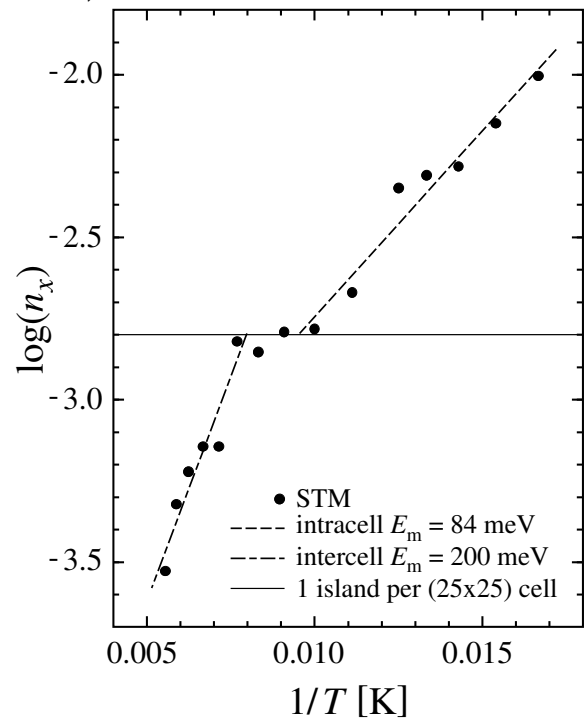

Fig. 13. Nucleation of a cluster superlattice on a dislocation network with a period of $7 \mathrm{~nm}$. (a) STM image of the network of $\langle 1 \overline{1} 0\rangle$-oriented partial dislocations formed by the second $\mathrm{Ag}$ monolayer on $\mathrm{Pt}(111)$ upon annealing to $800 \mathrm{~K}$. The inset shows a model of the trigonal strain relief pattern with its fcc- and hcp-stacking domains. (b) $\mathrm{Ag}$ nucleation on this network at $T=110 \mathrm{~K}$ yields an island superlattice $(\theta=0.10 \mathrm{ML})$. (c) Narrow size distributions are associated with the periodic island spacing (data as full symbols and binomial distribution as full line) as compared to nucleation on isotropic substrates (dashed line). (d) Arrhenius plot of experimental island densities showing the crossover in the rate limiting diffusion process from intracell diffusion to crossing of dislocations [149] 
and above the plateau give the activation energies for intracell and intercell diffusion, respectively. From the steeper slope of the latter the repulsive character of the dislocations becomes apparent. At the plateau intracell diffusion is fast enough for adatoms to visit the entire unit cell, although their thermal energy does not yet suffice to cross dislocations and exactly one island forms per unit cell.

In general there is a correlation between cluster spacings and cluster sizes [14]. Material deposited onto the area closest to an island is likely to attach to that island. This capture area of an island is its Voronoi polygon. For equidistant islands these areas are identical thus leading to very narrow island size distributions. In our example the Voronoi areas are the $(25 \times 25)$ network unit cells. The scaled island size distribution (see Fig. 13c) is accordingly significantly sharper than that obtained for homogeneous nucleation. We note that the measured standard deviation of $\sigma=0.20$ ( $\sigma$ equals approximately half the width at half maximum of the size distribution) represents an upper bound due to the residual width caused by STM-tip convolution. The theoretical lower bound for $\sigma$ is given by the case of ideal confinement of adatoms by infinite barriers. Then the cluster size distribution reduces to the statistics of deposition into the unit cells: for deposition of an average coverage $p$ into unit cells with size $n$ substrate atoms, the probability of finding $k$ atoms within a unit cell obeys a binomial distribution. This distribution, when normalized according to Fig. 13c, has a standard deviation of $\sigma=\sqrt{q / n p}$. For our example $(p=0.1, q=1-p=0.9$ and $n=625)$ this yields $\sigma=0.12$. This result from confined nucleation compares favorably to the best size distributions currently obtained in self-organized growth of quantum dots, which have $\sigma=0.16$ [160]. Due to reduced fluctuations $\sigma$ decreases for larger unit cells and larger coverages. For example deposition of $0.5 \mathrm{ML}$ into $(25 \times 25)$ unit cells leads to extremely sharp island size distributions with $\sigma=0.04$. These values refer to island areas, the distribution of island diameters having only half this width.

The prerequisites for confined nucleation are that dislocations must arrange themselves into periodic patterns, they must be repulsive for adatom diffusion, and there must be no exchange. The first condition is met by numerous heteroepitaxial systems. Well-ordered trigonal dislocation networks are found in a number of epitaxial metal $[38,145,146]$ and semiconductor systems [161]. The requirement for surface dislocations to be repulsive is found to be met by many metal systems. Exchange can be avoided by proper choice of the template. A Cu template formed on $\mathrm{Pt}(111)$ [162] was used instead of Ag to avoid exchange processes for the growth of Co and Fe arrays (see Fig. 14). In addition a low deposition temperature had to be used to avoid exchange (note that $\mathrm{Cu}(111)$ is unstable upon exchange with Co down to $170 \mathrm{~K}$ [163]) leading to the formation of many small clusters which were subsequently transferred into an ordered array by Ostwald ripening. Moiré structures [164-166] are also possible templates for self-organized growth of 


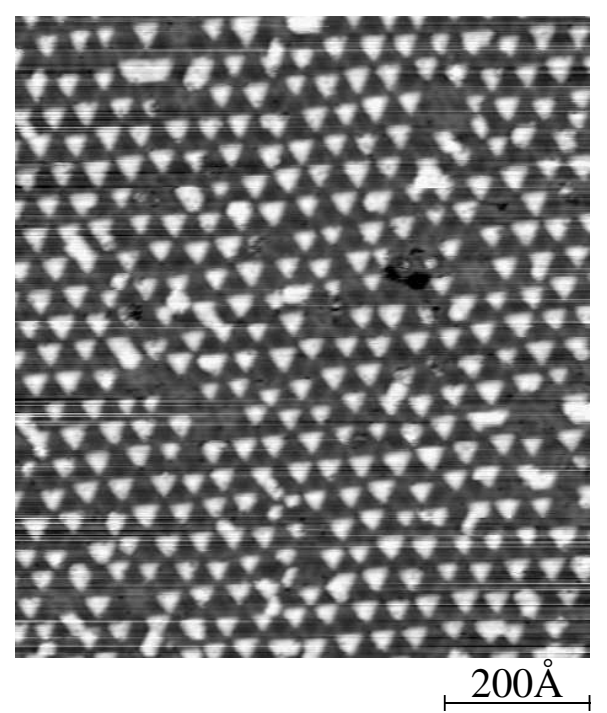

Fig. 14. STM image of a periodic array of $2 \mathrm{D}$ Fe islands (lattice constant $3.6 \mathrm{~nm}$ ) nucleated on the dislocation network of the $\mathrm{Cu}(13 \times 13)$ bilayer on $\mathrm{Pt}(111)$ (deposition at $60 \mathrm{~K}$, ripening at $250 \mathrm{~K})[149]$

cluster arrays. Similar to dislocation patterns they reveal periodic variations in adatom binding energy. In any of these cases the lattice constant of the array will be a fixed number given by the misfit of overlayer and substrate. However, the lattice constant of 2D alloy layers is a function of the composition and generally given by Vegard's law. Therefore the misfit and the supercell size are adjustable as has been shown for $\mathrm{Au}_{x} \mathrm{Ni}_{1-x}$-layers on $\mathrm{Ni}(111)$ [165]. This renders alloy layers interesting as templates for ordered nucleation.

We discussed two methods of self-organized growth of almost monodispersed, equally spaced nanostructures on substrates with periodic strain-relief patterns. Whereas the first relies on site specific exchange and risks being highly system specific, the second involving pure adatom diffusion seems to be more generally applicable. Since both methods require surface partial dislocations or Moiré structures that are known to form on hexagonal surfaces only, it is still a challenge to find a method for creating arrays with square symmetry.

\section{Single Crystal Oxide Surfaces}

The motivation for studying metal clusters on single crystal oxide supports is two-fold. The oxide support reduces electronic coupling with respect to metal or semiconductor surfaces, and metal clusters on oxide surfaces provide a model system for industrial supported catalysts [167]. (Such model catalysts can also be created by MOCVD [168] and by wet impregnation [169]). As in 
the case of metal/metal systems there is the need to achieve a detailed understanding of nucleation and growth in order to create the uniformity in cluster sizes and shapes required to draw conclusions from integral measurements of, e.g., reactivity and selectivity in a heterogeneous catalytic reaction. In the near future it is likely that the magnetism, electronic structure and superconductivity of clusters on weakly interacting supports will also be studied. Nucleation and growth of metals on oxides has been intensively studied in the past $[167,170-172]$. We focus in this section on two model cases with which we illustrate the role of substrate preparation, shed light on nucleation kinetics in the presence of attractive point defects characteristic of single-crystal oxide surfaces, and finally compare the information gained from local probes with that from Transmission Electron Microscopy (TEM).

a)

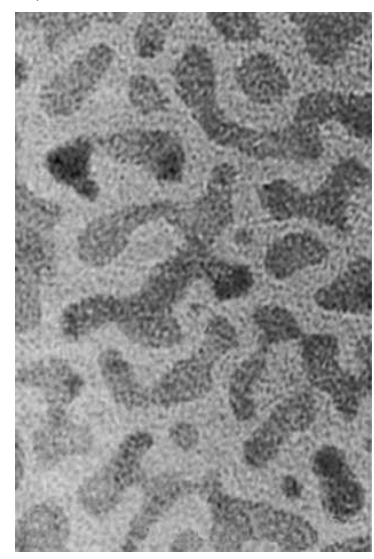

b)

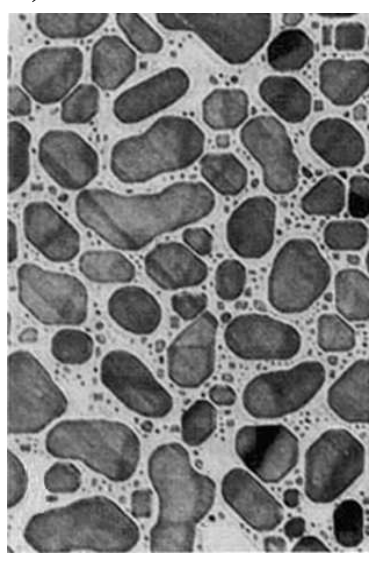

c)

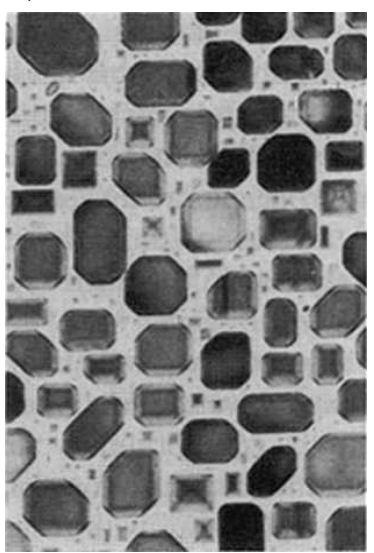

Fig. 15. TEM micrographs of $\mathrm{Pd}$ particles epitaxially grown on $\mathrm{MgO}(100)$ at (a) $300 \mathrm{~K},(\mathbf{b}) 433 \mathrm{~K}$, and (c) $673 \mathrm{~K}$, respectively $(\theta=5-10 \mathrm{ML})[173]$

The evolution of cluster shape with growth temperature is shown for the case of $\mathrm{Pd} / \mathrm{MgO}(100)$ in Fig. 15. The cluster shape at $300 \mathrm{~K}$ is $2 \mathrm{D}$ and the edges are rough, both of which are signatures for kinetically controlled clusters. Upon deposition at $673 \mathrm{~K}$ clusters are truncated half-octahedrons. This cluster shape is considered to be the thermodynamic equilibrium shape since the height/base ratio was found to be independent of cluster size [173]. The cluster shape as a Wulff polyhedron gives access to the surface free energies of the respective facets. For the equilibrium shape to be reached the adatoms deposited onto the substrate $(\theta=5-10 \mathrm{ML})$ have to be able to climb up to the cluster tops where they are more strongly bound than on $\mathrm{MgO}(100)$. However, equilibrium is only reached for isolated clusters, while 
for the coalesced clusters, deviations from the equilibrium shape show that the system is still to some extent affected by kinetic limitations.

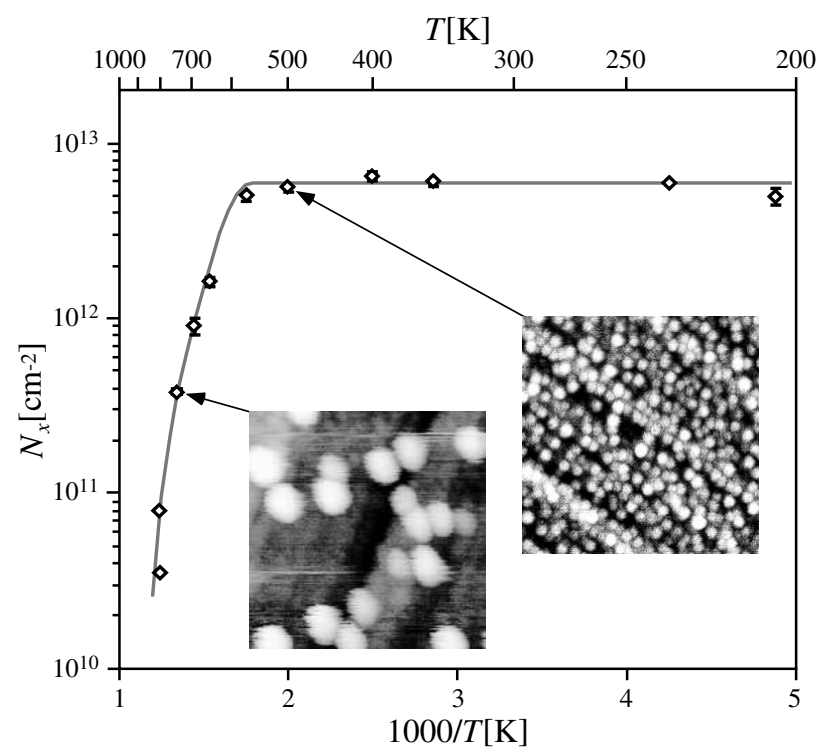

Fig. 16. Arrhenius plot of island densities for Pd deposits on Ar-cleaved $\mathrm{MgO}(100)$ obtained with non-contact AFM (size of images $1000 \times 1000 \AA$ ) [174]. The solid line is a mean-field rate equation model accounting for defect trapping $(\theta=0.1 \mathrm{ML}$, $\left.F=2.7 \times 10^{-4} \mathrm{ML} / \mathrm{s}\right)$. See text for model parameters

The cluster density is critically dependent on the preparation of the single crystal $\mathrm{MgO}(100)$ surface. Cleavage in air and subsequent evaporation under UHV conditions yields a 10-times larger island density than UHV cleavage and in situ deposition [167]. This is indicative of heterogeneous nucleation at defects created upon exposure to the ambient atmosphere. On the other hand, cleavage in Ar atmosphere with subsequent annealing in oxygen $(750 \mathrm{~K}$, $p_{\mathrm{O}_{2}}=1 \times 10^{-4}$ mbar) gives the same densities as UHV cleavage [174]. Information concerning nucleation kinetics for $\mathrm{Pd}$ on $\mathrm{MgO}(100)$ was obtained from average cluster densities deduced from VT-AFM images taken as a function of deposition temperature [174]. The AFM images reproduced in Fig. 16 show that cluster nucleation takes place at terraces and only occasionally at steps. On the other hand, the Arrhenius plot of the island density is clear evidence for heterogeneous nucleation at defects with large trapping energies. The trapping defects must thus be located at substrate terraces. There are ab initio calculations investigating the $\mathrm{Pd}$ trapping energies of several possible defects on $\mathrm{MgO}(100)$ terraces; one which seems likely to be involved is an oxygen vacancy, the so-called neutral $F_{\mathrm{s}}$-center [175]. 
Comparing experimental data with calculations using mean-field nucleation theory including trapping defects (solid line in Fig. 16), the relevant system parameters could be derived. The diffusion energy on the defect-free $\mathrm{MgO}(100)$ terraces must be rather low $\left(E_{\mathrm{m}} \leq 0.2 \mathrm{eV}\right)$ for all the Pd atoms to reach the defect sites down to $T=200 \mathrm{~K}$. The length of the plateau towards higher $T$ defines the minimum trapping energy, $E_{\mathrm{t}} \geq 1.2 \mathrm{eV}$. This bound compares reasonably well with the theoretical value of $E_{\mathrm{t}}=1.55 \mathrm{eV}$ [175]. The knee at $600 \mathrm{~K}$ is best fitted by a transition from $i=1$ to $i=3$, i.e., traps remain populated by one $\mathrm{Pd}$ atom, but the second and third bound to it break up while only 4 atoms represent a stable cluster at a trap. The deduced lateral bond energy of $E_{\mathrm{b}}=1.2 \mathrm{eV}$ lies slightly below the theoretical gas phase value which is reasonable on a weakly bonding substrate. Incomplete condensation starts at $750 \mathrm{~K}$, as evidenced by the decrease in sticking deduced from AES measurements, and by the final increase in the slope of $\log \left(n_{x}\right)$ vs. $1 / T$, obtained in the model for an adsorption energy of $E_{\mathrm{a}}=1.2 \mathrm{eV}$. The parameter set derived for the model system Pd/MgO(100) constitutes a valid basis for comparison with theory and is a step towards a quantitative understanding of nucleation and growth in metal/insulator systems.

The imaging of clusters with local probes enables in situ studies and gives access to the cluster height. However, the convolution with the tip falsifies absolute values of the projected surface area, which are reliably obtained by ex situ TEM. In our example of $\mathrm{Pd} / \mathrm{MoS}_{2}$ the convolution is clearly visible since clusters appear much larger in the STM topograph than they are in reality (see Fig. 17). Accordingly the size distribution derived from STM is centered at larger average sizes. The effect of tip convolution is expected to be even more dramatic for AFM images of metal clusters, e.g., on $\mathrm{MgO}$. Such images have to be taken in non-contact mode to prevent displacement of the weakly bound clusters by the tip-sample interaction forces appearing in contact mode. Awareness of the convolution effect when deriving absolute cluster sizes and size distributions from local probe techniques is therefore important.

We end our section about metals on insulators by discussing a method that reduces the width of the cluster size distribution for these systems. It is based on the fact that the optical absorption coefficient of metal particles is strongly size dependent. Laser irradiation of clusters (Ag on a quartz support) with a frequency chosen in resonance with the plasmon modes for a certain cluster size selectively heats these clusters which then evaporate atoms and shrink in size. Specific cluster sizes can thereby be reduced or even totally removed. Successive irradiation with two laser wavelengths removes the smallest clusters and causes a size reduction of the largest ones. Thus both tails of the size distribution are removed which reduces its width (to $\sigma \sim 0.26$ ), as verified by means of AFM and optical extinction spectra [177]. In the sense of leading to better defined cluster sizes the method is 
a)

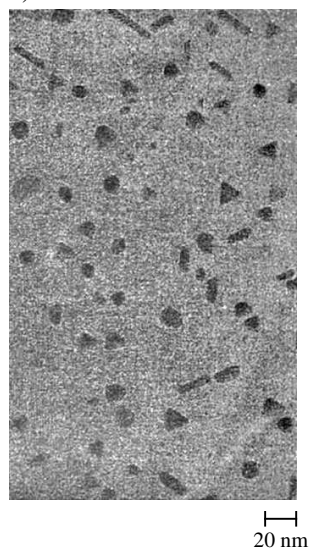

c)

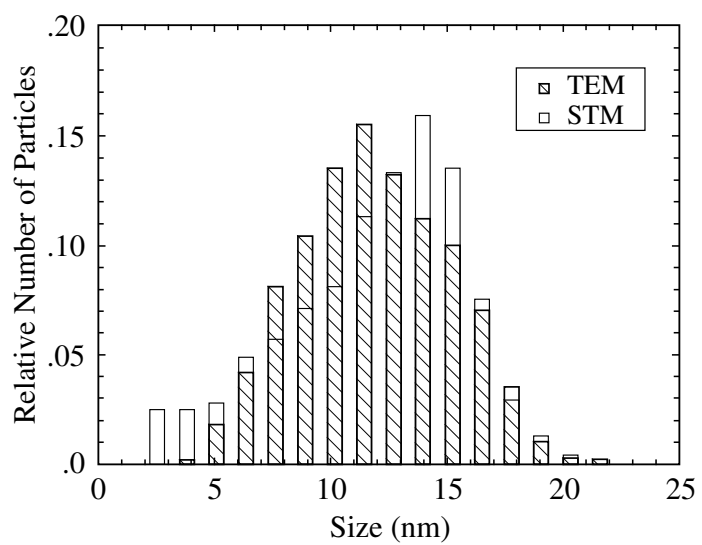

b)

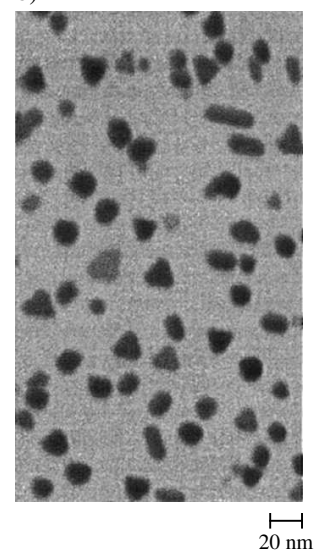

$\mathrm{nm}$

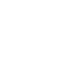


basic science and applications towards objects with smaller length scales. Questions that may be addressed in the near future are chemical reactivity, transport, and magnetic properties of surface supported clusters evolving from the quantum confinement of electrons in the clusters. The advantage of cluster growth with respect to atomic manipulation with local probes is the high cluster density enabling access to the clusters' chemical and physical properties with integrating experimental techniques. In order to get the relevant information from integrating measurements, however, size and shape

uniformity are essential, requiring detailed understanding and control of the atomic processes underlying cluster growth at surfaces.

\section{References}

1. Growth and Properties of Ultrathin Epitaxial Layers, Vol. 8 of The Chemical Physics of Solid Surfaces and Heterogeneous Catalysis, edited by D. A. King and D. P. Woodruff (Elsevier Science, Amsterdam, 1997).

2. E. Bauer, Z. Kristallographie 110, 372 (1958).

3. M. J. Stowell, Phil. Mag. 26, 349 (1972).

4. J. A. Venables, Philos. Mag. 17, 697 (1973).

5. B. Lewis and J. C. Anderson, Nucleation and Growth of Thin Films (Academic Press, New York, San Francisco, London, 1978).

6. G. Ehrlich, Surf. Sci. 246, 1 (1991).

7. G. Ehrlich, Appl. Phys. A 55, 403 (1992).

8. G. L. Kellogg, Surf. Sci. Rep. 21, 1 (1994).

9. T. T. Tsong, Atom-probe field ion microscopy (Cambridge University Press, New York, 1990).

10. H. Brune, Surf. Sci. Rep. 31, 121 (1998).

11. M. C. Bartelt, M. C. Tringides, and J. W. Evans, Phys. Rev. B 47, 13891 (1993).

12. J. G. Amar and F. Family, Phys. Rev. Lett. 74, 2066 (1995).

13. M. C. Bartelt and J. W. Evans, Phys. Rev. B 54, R17359 (1996).

14. P. A. Mulheran and J. A. Blackman, Phys. Rev. B 53, 10261 (1996).

15. S. C. Wang and G. Ehrlich, J. Chem. Phys. 94, 4071 (1991).

16. A. Hitzke, M. B. Hugenschmidt, and R. J. Behm, Surf. Sci. 389, 8 (1997).

17. S. Günther, A. Hitzke, and R. J. Behm, Surf. Rev. and Lett. 4, 1103 (1997).

18. H. Brune et al., Phys. Rev. B 60, 5991 (1999).

19. H. Brune, J. Wintterlin, R. J. Behm, and G. Ertl, Phys. Rev. Lett. 68, 624 (1992).

20. J. Wintterlin, R. Schuster, and G. Ertl, Phys. Rev. Lett. 77, 123 (1996).

21. G. Vandoni et al., Surf. Sci. 320, L63 (1994).

22. S. C. Wang and G. Ehrlich, Phys. Rev. Lett. 70, 41 (1993).

23. S. C. Wang and G. Ehrlich, Phys. Rev. Lett. 71, 4174 (1993).

24. G. L. Kellogg, Phys. Rev. Lett. 76, 98 (1996).

25. A. F. Voter and J. D. Doll, J. Chem. Phys. 80, 5832 (1984).

26. R. Gomer, Rep. Prog. Phys. 53, 917 (1990).

27. G. Ehrlich and F. Watanabe, Langmuir 7, 2555 (1991).

28. F. Watanabe and G. Ehrlich, J. Chem. Phys. 96, 3191 (1992). 
29. T. A. Witten and L. M. Sander, Phys. Rev. Lett. 47, 1400 (1981).

30. P. Meakin, in Phase Tansitions and Critical Phenomena, edited by C. Domb and J. L. Lebowitz (Academic Press, New York, 1988), Vol. 12, p. 335.

31. A. L. Barabási and H. E. Stanley, Fractal concepts in surface growth (Cambridge University Press, New York, 1995).

32. G. Ehrlich and F. G. Hudda, J. Chem. Phys. 44, 1039 (1966).

33. R. L. Schwoebel and E. J. Shipsey, J. Appl. Phys. 37, 3682 (1966).

34. R. Stumpf and M. Scheffler, Phys. Rev. Lett. 72, 254 (1994).

35. B. D. Yu and M. Scheffler, Phys. Rev. Lett. 77, 1095 (1996).

36. P. J. Feibelman, Phys. Rev. Lett. 81, 168 (1998).

37. G. Ehrlich, Surf. Sci. 331-333, 865 (1995).

38. J. A. Meyer et al., Phys. Rev. B 51, 14790 (1995).

39. P. Smilauer and S. Harris, Phys. Rev. B 51, 14798 (1995).

40. K. Bromann, H. Brune, H. Röder, and K. Kern, Phys. Rev. Lett. 75, 677 (1995).

41. K. Morgenstern et al., Phys. Rev. Lett. 80, 556 (1998).

42. I. Markov, Phys. Rev. B 56, 12544 (1997).

43. K. R. Roos, R. Bhutani, and M. C. Tringides, Surf. Sci. 384, 62 (1997).

44. C. M. Zhang et al., J. Cryst. Growth 174, 851 (1997).

45. J. Alvarez, E. Lundgren, X. Torrelles, and S. Ferrer, Phys. Rev. B 57, 6325 (1998).

46. J. A. Venables, Phys. Rev. B 36, 4153 (1987).

47. S. Liu, L. Bönig, and H. Metiu, Phys. Rev. B 52, 2907 (1995).

48. M. C. Bartelt et al., Phys. Rev. B 53, 4099 (1996).

49. H. Brune, H. Röder, C. Boragno, and K. Kern, Phys. Rev. Lett. 73, 1955 (1994).

50. J. A. Venables, G. D. T. Spiller, and M. Hanbücken, Rep. Prog. Phys. 47, 399 (1984).

51. Y. W. Mo, J. Kleiner, M. B. Webb, and M. G. Lagally, Phys. Rev. Lett. 66, 1998 (1991).

52. J. A. Stroscio, D. T. Pierce, and R. A. Dragoset, Phys. Rev. Lett. 70, 3615 (1993).

53. M. Bott et al., Phys. Rev. Lett. 76, 1304 (1996).

54. B. Müller et al., Phys. Rev. B 54, 17858 (1996).

55. G. S. Bales and D. C. Chrzan, Phys. Rev. B 50, 6057 (1994).

56. H. Brune et al., Phys. Rev. B 52, R14380 (1995).

57. C. Ratsch and M. Scheffler, Phys. Rev. B 58, 13163 (1998).

58. B. Fischer et al., Phys. Rev. Lett. 82, 1732 (1999).

59. J. V. Barth et al., Phys. Rev. Lett. in press (1999).

60. T. B. Grimley, Proc. Phys. Soc. 90, 751 (1967).

61. M. C. Bartelt and J. W. Evans, Phys. Rev. B 46, 12675 (1992).

62. L. H. Tang, J. Phys. (Paris) 13, 935 (1993).

63. C. Ratsch, A. Zangwill, P. Smilauer, and D. D. Vvedensky, Phys. Rev. Lett. 72, 3194 (1994).

64. J. A. Stroscio and D. T. Pierce, Phys. Rev. B 49, 8522 (1994).

65. T. Vicsek, Fractal Growth Phenomena (World Scientific, Signapore, 1989).

66. H. Takayasu, Fractals in the physical sciences (Manchester University Press, Manchester, New York, 1990).

67. Y. Furukawa, Chemie in unserer Zeit 2, 58 (1997). 
68. R. Q. Hwang, J. Schröder, C. Günther, and R. J. Behm, Phys. Rev. Lett. 67, 3279 (1991).

69. H. Röder et al., Nature 366, 141 (1993).

70. H. Brune, C. Romainczyk, H. Röder, and K. Kern, Nature 369, 469 (1994).

71. T. Michely, M. Hohage, M. Bott, and G. Comsa, Phys. Rev. Lett. 70, 3943 (1993).

72. H. Brune et al., Surf. Sci. 349, L115 (1996).

73. Y. Couder, N. Gérard, and M. Rabaud, Phys. Rev. A 34, 5175 (1986).

74. A. Pimpinelli, J. Villain, and D. E. Wolf, J. Phys. (Paris) 3, 447 (1993).

75. M. C. Bartelt and J. W. Evans, Surf. Sci. 314, L829 (1994).

76. G. S. Bales and D. C. Chrzan, Phys. Rev. Lett. 74, 4879 (1995).

77. H. Röder, K. Bromann, H. Brune, and K. Kern, Phys. Rev. Lett. 74, 3217 (1995).

78. M. Hohage et al., Phys. Rev. Lett. 76, 2366 (1996).

79. F. B. de Mongeot et al., Surf. Sci. 411, 249 (1998).

80. P. Stoltze, J. Phys. Condens. Matter 6, 9495 (1994).

81. P. Ruggerone, C. Ratsch, and M. Scheffler, in Growth and Properties of Ultrathin Epitaxial Layers, edited by D. A. King and D. P. Woodruff (Elsevier Science, Amsterdam, 1997), Vol. 8, p. 490.

82. C. L. Liu, Surf. Sci. 316, 294 (1994).

83. D. D. Chambliss and K. E. Johnson, Phys. Rev. B 50, 5012 (1994).

84. J. A. Stroscio, D. T. Pierce, and R. A. Dragoset, Phys. Rev. Lett. 70, 3615 (1993).

85. E. Kopatzki, S. Günther, W. Nichtl-Pecher, and R. J. Behm, Surf. Sci. 284, 154 (1993).

86. E. Hahn, E. Kampshoff, N. Wälchli, and K. Kern, Phys. Rev. Lett. 74, 1803 (1995).

87. J. M. Wen et al., Phys. Rev. Lett. 76, 652 (1996).

88. Z. Zhang, X. Chen, and M. G. Lagally, Phys. Rev. Lett. 73, 1829 (1994).

89. B. Müller et al., Phys. Rev. Lett. 80, 2642 (1998).

90. J. K. Nørskov, K. W. Jacobsen, P. Stoltze, and L. B. Hansen, Surf. Sci. 283, 277 (1993).

91. P. Feibelman, Phys. Rev. B 60, 4972 (1999).

92. C. Günther et al., Ber. Bunsenges. Phys. Chem. 97, 522 (1993).

93. K. Bromann, H. Brune, M. Giovannini, and K. Kern, Surf. Sci. 388, L1107 (1997).

94. K. Bromann, H. Brune, and K. Kern, unpublished results (1997).

95. M. C. Bartelt and J. W. Evans, Surf. Sci. 314, L829 (1994).

96. A. Bogicevic et al., Phys. Rev. B 57, R9459 (1998).

97. R. Stumpf and M. Scheffler, Phys. Rev. B 53, 4958 (1996).

98. A. Bogicevic, J. Strömquist, and B. I. Lundquist, Phys. Rev. Lett. 81, 637 (1998).

99. R. Q. Hwang et al., J. Vac. Sci. Technol. A 10, 1970 (1992).

100. J. Vrijmoeth et al., in Magnetism and Structure in Systems of Reduced Dimension, edited by R. F. C. Farrow (Plenum Press, New York, 1993), p. 55.

101. C. Boragno, H. Röder, H. Brune, and K. Kern, to be published (1999).

102. B. Voigtländer, G. Meyer, and N. M. Amer, Surf. Sci. 255, L529 (1991).

103. J. A. Stroscio, D. T. Pierce, R. A. Dragoset, and P. N. First, J. Vac. Sci. Technol. A 10, 1981 (1992). 
104. T. Michely and G. Comsa, Surf. Sci. 256, 217 (1991).

105. T. Michely, M. Hohage, M. Bott, and G. Comsa, Phys. Rev. Lett. 70, 3943 (1993).

106. M. Kalff, G. Comsa, and T. Michely, Phys. Rev. Lett. 81, 1255 (1998).

107. S. Liu, Z. Zhang, G. Comsa, and H. Metiu, Phys. Rev. Lett. 71, 2967 (1993).

108. J. Jacobsen, K. W. Jacobsen, and J. K. Nørskov, Surf. Sci. 359, 37 (1996).

109. C. Ratsch, P. Ruggerone, and M. Scheffler, in Surface Diffusion: Atomistic and Collective Processes, edited by M. C. Tringides (Plenum Press, New York, 1997), Vol. 360, p. 83.

110. S. Ovesson, A. Bogicevic, and B. L. Lundqvist, Phys. Rev. Lett. 83, 2608 (1999).

111. M. Kalff, Ph.D. thesis, Forschungszentrum Jülich, 1999.

112. S. Horch et al., Nature 398, 134 (1999).

113. F. Besenbacher, personal communication (1999).

114. Physics and Chemistry of Finite Systems: From Clusters to Crystals, edited by P. Jena, S. N. Khanna, and B. K. Rao (Kluwer, Dordrecht, 1992).

115. W. Ostwald, Z. Phys. Chem. (Leipzig) 34, 495 (1900).

116. C. Wagner, Z. Elektrochem. 65, 581 (1961).

117. L. M. Lifshitz and V. V. Slyozov, J. Phys. Chem. Solids 19, 35 (1961).

118. K. Morgenstern, G. Rosenfeld, and G. Comsa, Phys. Rev. Lett. 76, 2113 (1996).

119. W. Theis, N. C. Bartelt, and R. M. Tromp, Phys. Rev. Lett. 75, 3328 (1995).

120. P. Blandin, C. Massobrio, and P. Ballone, Phys. Rev. Lett. 72, 3072 (1994).

121. H. Röder, H. Brune, and K. Kern, Phys. Rev. Lett. 73, 2143 (1994).

122. P. M. Petroff, A. C. Gossard, and W. Wiegmann, Appl. Phys. Lett. 45, 620 (1984).

123. Y. W. Mo and F. J. Himpsel, Phys. Rev. B 50, 7868 (1994).

124. J. Shen et al., Phys. Rev. B 56, 2340 (1997).

125. V. Marsico, M. Blanc, K. Kuhnke, and K. Kern, Phys. Rev. Lett. 78, 94 (1997).

126. M. Blanc, K. Kuhnke, V. Marsico, and K. Kern, Surf. Sci. 414, L964 (1998).

127. P. Gambardella et al., Phys. Rev. B in press (1999).

128. M. A. v. Hove et al., Surf. Sci. 103, 189 (1981).

129. V. Fiorentini, M. Methfessel, and M. Scheffler, Phys. Rev. Lett. 71, 1051 (1993).

130. S. Günther et al., Phys. Rev. Lett. 73, 553 (1994).

131. L. Bönig, S. Liu, and H. Metiu, Surf. Sci. 365, 87 (1996).

132. T. R. Linderoth et al., Phys. Rev. Lett. 77, 87 (1996).

133. J. J. Mortensen et al., Surf. Sci. 400, 290 (1997).

134. T. R. Linderoth et al., Phys. Rev. Lett. 78, 4978 (1997).

135. F. Montalenti and R. Ferrando, Phys. Rev. B 58, 3617 (1998).

136. M. B. Hugenschmidt, M. Ruff, A. Hitzke, and R. J. Behm, Surf. Sci. 388, L1100 (1997).

137. J. P. Bucher et al., Europhys. Lett. 27, 473 (1994).

138. N. Waelchli, Ph.D. thesis, Ecole Polytechnique Fédérale de Lausanne, 1997.

139. J. V. Barth, personal communication (1999).

140. Y. Li et al., Phys. Rev. B 56, 12539 (1997).

141. R. Ferrando, F. Hontinfinde, and A. C. Levi, Phys. Rev. B 56, R4406 (1997). 
142. C. Mottet, R. Ferrando, F. Hontinfinde, and A. C. Levi, Surf. Sci. 417, 220 (1998).

143. C. Pearson, M. Krueger, and E. Ganz, Phys. Rev. Lett. 76, 2306 (1996).

144. H. Brune, H. Röder, C. Boragno, and K. Kern, Phys. Rev. B 49, 2997 (1994).

145. C. Günther, J. Vrijmoeth, R. Q. Hwang, and R. J. Behm, Phys. Rev. Lett. 74, 754 (1995).

146. H. Brune and K. Kern, in Growth and Properties of Ultrathin Epitaxial Layers, edited by D. A. King and D. P. Woodruff (Elsevier Science, Amsterdam, 1997), Vol. 8, p. 149.

147. J. A. Meyer, P. Schmid, and R. J. Behm, Phys. Rev. Lett. 74, 3864 (1995).

148. T. Michely, M. Hohage, S. Esch, and G. Comsa, Surf. Sci. 349, L89 (1996).

149. H. Brune, M. Giovannini, K. Bromann, and K. Kern, Nature 394, 451 (1998).

150. J. V. Barth, H. Brune, G. Ertl, and R. J. Behm, Phys. Rev. B 42, 9307 (1990).

151. D. D. Chambliss, R. J. Wilson, and S. Chiang, Phys. Rev. Lett. 66, 1721 (1991).

152. J. A. Meyer, J. D. Baikie, E. Kopatzki, and R. J. Behm, Surf. Sci. 365, L647 (1996).

153. B. Voigtländer, G. Meyer, and N. M. Amer, Phys. Rev. B 44, 10354 (1991).

154. S. Padovani, P. Molinás-Mata, F. Scheurer, and J. P. Bucher, Appl. Phys. A 66, S1199 (1998).

155. E. I. Altman and R. J. Colton, Surf. Sci. 304, L400 (1994).

156. D. D. Chambliss and R. J. Wilson, J. Vac. Sci. Technol. B 9, 928 (1991).

157. M. A. Krzyzowski, Ph.D. thesis, Universität Bonn, 1995.

158. B. Fischer et al., Surf. Sci. 389, 366 (1997).

159. C. Ratsch, A. P. Seitsonen, and M. Scheffler, Phys. Rev. B 55, 6750 (1997).

160. S. Fafard et al., Phys. Rev. B 52, 5752 (1995).

161. M. H. von Hoegen et al., Surf. Sci. 298, 29 (1993).

162. B. Holst, M. Hohlen, K. Wandelt, and W. Allison, Surf. Sci. 377-379, 891 (1997).

163. M. Ø. Pedersen et al., Surf. Sci. 387, 86 (1997).

164. T. Wiederholt et al., Surf. Sci. 324, 91 (1994).

165. F. Besenbacher, L. P. Nielsen, and P. T. Sprunger, in Growth and Properties of Ultrathin Epitaxial Layers, edited by D. A. King and D. P. Woodruff (Elsevier, Amsterdam, 1997), Vol. 8, p. 207.

166. M. Böhringer et al., Surf. Sci. 367, 245 (1996).

167. C. R. Henry, Surf. Sci. Rep. 31, 231 (1998).

168. S. K. Purnell, X. Xu, D. W. Goodman, and B. C. Gates, J. Phys. Chem. 98, 4076 (1994).

169. M. Valden et al., Surf. Sci. 307-309, 193 (1994).

170. H. Poppa, Catal. Rev. Sci. Eng. 35, 359 (1993).

171. R. J. Lad, Surf. Rev. Lett. 2, 109 (1995).

172. C. T. Champbell, Surf. Sci. Rep. 27, 1 (1997).

173. C. Henry, C. Chapon, S. Giorgio, and C. Goyhenex, in Chemisorption, Reactivity of Clusters, Thin Films, edited by R. M. Lambert and G. Pacchioni (Kluwer, Dordrecht, 1997), p. 117.

174. G. Haas et al., Phys. Rev. B submitted for publication (1999).

175. A. M. Ferrari and G. Pacchioni, J. Phys. Chem. 100, 9032 (1996).

176. E. Perrot, Ph.D. thesis, Université de Marseille, 1996.

177. J. Bosbach et al., Appl. Phys. Lett. 74, 2605 (1999). 